\title{
On the non-optimality of tree structures for heat conduction
}

\author{
Yan, Suna ; Wang, Fengwen; Sigmund, Ole
}

Published in:

International Journal of Heat and Mass Transfer

Link to article, DOI:

10.1016/j.ijheatmasstransfer.2018.01.114

Publication date:

2018

Document Version

Peer reviewed version

Link back to DTU Orbit

Citation (APA):

Yan, S., Wang, F., \& Sigmund, O. (2018). On the non-optimality of tree structures for heat conduction. International Journal of Heat and Mass Transfer, 122, 660-680.

https://doi.org/10.1016/j.ijheatmasstransfer.2018.01.114

\section{General rights}

Copyright and moral rights for the publications made accessible in the public portal are retained by the authors and/or other copyright owners and it is a condition of accessing publications that users recognise and abide by the legal requirements associated with these rights.

- Users may download and print one copy of any publication from the public portal for the purpose of private study or research.

- You may not further distribute the material or use it for any profit-making activity or commercial gain

- You may freely distribute the URL identifying the publication in the public portal

If you believe that this document breaches copyright please contact us providing details, and we will remove access to the work immediately and investigate your claim. 


\title{
On the non-optimality of tree structures for heat conduction
}

\author{
Suna Yan ${ }^{\mathrm{a}, *}$, Fengwen Wang ${ }^{\mathrm{b}}$, Ole Sigmund ${ }^{\mathrm{b}}$ \\ ${ }^{a}$ School of Mechanical Engineering, State Key Laboratory for Manufacturing Systems Engineering, Xi'an Jiaotong \\ University, $710049 \mathrm{Xi}$ 'an, China \\ ${ }^{b}$ Department of Mechanical Engineering, Solid Mechanics, Technical University of Denmark, 2800 Kgs. Lyngby, \\ Denmark
}

\begin{abstract}
This paper revisits topology optimization of heat conduction structures for minimum thermal compliance and minimum maximum temperature, respectively. For both optimization problems, volume-to-line and volume-to-point structures are optimized based on three material interpolation models describing different design spaces regarding the relation between material density and effective conductivity. The numerical results are backed up by analytical studies. Comparisons of results show that lamellar needle structures, rather than commonly seen tree structures, constitute the optimal topologies for heat conduction. This contradicts the usual hypothesis drawn from the observation of natural transferring systems and designs from numerous related studies. The conclusion still holds when a minimum length scale is imposed for both high and low conductive phases. Finally, the minimum thermal compliance problem and the min-max temperature problem are compared in terms of optimal microstructures. Lamellar microstructures with the normal to the material layers bisecting the gradients of direct and adjoint solutions are optimal for both types of problems. The variable thickness sheet model is optimal only for the self-adjoint minimum thermal compliance problem.
\end{abstract}

Keywords: Heat conduction, Volume-to-point problem, Tree structures, Lamellar needle structures, Topology optimization

\footnotetext{
${ }^{*}$ Corresponding author

Email address: sunayan.me@gmail.com (Suna Yan)

This work was carried out while Suna Yan was a visiting student at Department of Mechanical Engineering, Technical University of Denmark.
} 


\section{Introduction}

The volume-to-point problem in heat conduction has attracted considerable attention. A general definition of the problem based on the concept proposed by Bejan [1] is as follows: Consider a finite-size volume in which heat is generated at every point. The volume is cooled through a small 5 patch (heat sink) located on its boundary where the temperature is prescribed. Every point of the volume is made of either high or low conductive material and the volume fraction of high conductive material is finite. Design the distribution of materials such that the heat conduction performance of the volume is maximized. In the extensive literature on this problem, different parameters are used as the metric for the heat conduction performance and different design approaches are proposed, as summarized in Table 1 .

Table 1: Literature on the volume-to-point problem

\begin{tabular}{|c|c|c|c|}
\hline Objective function (min) & Approach & Rule & Paper \\
\hline$T_{\max }$ & Constructal theory & Theoretical analysis & 17 \\
\hline$C_{\mathrm{th}}$ & TO (Density-based models) & MMA & 8 \\
\hline$T_{\max }, T_{\mathrm{av}}, C_{\mathrm{th}}$ & TO (SIMP) & MMA & 9 \\
\hline Weighted sum of $T_{\max }, T_{\mathrm{av}}$ & TO (SIMP) & MMA & 10 \\
\hline$C_{\text {th }}$ & TO (SIMP) & MMA & 11,12 \\
\hline$C_{\mathrm{th}}$ & TO (SIMP) & Optimality criterion & 13 \\
\hline Weighted sum of $C_{\mathrm{th}}, E_{\mathrm{in}}$ & TO (Level set) & & 14 \\
\hline$C_{\mathrm{th}}$ & TO (Microstructures) & MMA & 15 \\
\hline$C_{\mathrm{th}}$ & TO (SSM and SIMP) & MMA & 16 \\
\hline Geometric $T_{\mathrm{av}}$ & TO & MMA & 17 \\
\hline Heat flow path & TO & Evolutionary algorithm & 18 \\
\hline Entropy generation & TO & $\begin{array}{l}\text { Temperature gradient- } \\
\text { based principle }\end{array}$ & 19 \\
\hline \multirow{4}{*}{$\begin{array}{l}T_{\max }, T_{\mathrm{av}} \\
\text { Temperature gradient } \\
\text { difference } \\
T_{\mathrm{av}}\end{array}$} & TO & Genetic algorithm & 20,21 \\
\hline & TO & Cellular automaton & 22 \\
\hline & Generative approach & Growth algorithm & 23 \\
\hline & Generative approach & $\begin{array}{l}\text { Space colonization algorithm } \\
\text { and genetic algorithm }\end{array}$ & 24 \\
\hline$T_{\max }$ & Generative approach & Genetic algorithm & 25 \\
\hline
\end{tabular}

$T_{\max }:$ maximum temperature, $T_{\mathrm{av}}$ : average temperature, $C_{\mathrm{th}}:$ thermal compliance, $E_{\mathrm{in}}:$ interface energy, TO: topology optimization, SIMP: Solid Isotropic Material with Penalization model, SSM: Stiffness Spreading Method.

At the same time as proposing the volume-to-point problem, Bejan developed the constructal theory [1] to solve it by which material distributions minimizing the maximum temperature were 
theoretically determined. The smallest building blocks (elemental volumes) were optimized at first in terms of the geometric aspect ratio. To cool a greater volume, elemental volumes were lined up by a new high conductive path and the first assembly was built. Higher order assemblies were assembled successively from lower order ones in a similar way. Although geometric parameters of constructal networks were optimized step by step, no explanation was given on the optimality of the assembling approach which inevitably leads to trees with branches. Later, more exact constructal theory-based derivations were developed in [4] by dropping the assumption on the effective conductivity of the first assembly used in [1. A new series of constructal trees were obtained, but still left the question on the optimality of the assembling approach unresolved. In [3], Bejan concluded that increasing the complexity of constructal trees makes sense for reducing the maximum temperature of the volume. However, Ghodoossi [5, 6] proved that the sequence of constructal designs from simple toward complex trees does not improve the heat conduction performance in general. Marck et al. [7. found that the performance of construtal trees strongly depends on the volume fraction of the high conductive material and not on the structural complexity.

Different from the constructal theory, topology optimization methods provide more degrees of freedom in design and allow to study different objective functions and complex design domains [26]. As shown in Table 1, thermal compliance, maximum temperature and weighted sums of them are the most widely used objective functions. Design approaches employing the SIMP model [27, 28], level-set and microstructures have been studied. Gradient-based algorithms and genetic algorithms are used to solve the optimization problems. To explore the effect of design space, Manuel and Lin [8] used 6 fixed and 6 tunable density-based material interpolation models, including the Solid Isotropic Material with Penalization (SIMP) model and the Rational Approximation of Material Properties (RAMP) model 29, for minimizing the thermal compliance. The design results from a uniform initial guess include gray material distributions and discrete trees. In traditional level-set based topology optimization approaches, holes cannot be added to the design domain and thus initial guesses have a crucial effect on design results. To circumvent this problem, Yamada et al. [14 incorporated a fictitious interface energy term into the thermal compliance and reformulated the level set function. The obtained designs for the volume-to-point problem are trees with different degrees of geometry complexity controlled by the weight of the fictitious interface energy term. Li et al. [16] studied the optimization of volume-to-point structures for high and low conduction regions with the addition of high conductive pipes, also resulting in tree-like structures. Changing 
implementation details of the optimization process leads to different design results despite starting from the same initial guess, which exemplifies the existence of local minima. In [18, Li et al. proposed a concept named heat flow path across which no heat is transferred and thus the heat flow paths are tangent to temperature gradients at every point of the design domain. During the optimization, the distribution of heat flow paths are homogenized by converting elements at low heat flux regions to be low conductive. Different tree-like design results are obtained from different initial guesses.

In the references listed in Table 1 employing topology optimization approaches, all the resulting discrete optimized designs are tree structures with only two exceptions. Based on single precision graphical processing unit (GPU) programming, Wadbro and Berggren [13 optimized a volumeto-point structure using up to one million elements. In addition, a strict convergence condition and a slow continuation approach to update the penalization factor were used. Apparently, in this case the optimized designs became lamellar or needle-like instead of tree structures, even if based on a uniform initial guess. By construction, due to an anisotropic formulation not un-like the microstructure approach used in our work, Dede [15] also obtained lamellar structures instead of trees, but without a density dependence.

Probably inspired by the functional similarity between heat transfer structures and natural mass transfer systems and the branching configurations possessed by the latter, some generative approaches have been developed based on the assumption that dendritic or tree-like topologies are preferable for heat conduction. A volume-to-point problem similar to that in [16] was studied in [23, where high conductive pipes were added into the low conductive base material step-by-step and positions and sizes of high conductive pipes were optimized. Aiming at obtaining branching configurations of high conductive pipes which were conceived as optimal for heat conduction, a branching promotion strategy was developed. The resulting designs are inevitably tree structures. In [24, the distribution of high conductive material was parameterized as the distribution of auxins and extracted by a space colonization algorithm which was chosen specifically to produce complex dendritic structures. By updating the auxin distribution, diversified dendritic topologies were obtained. In [25], the lengths, thicknesses and bifurcation angles of dendritic structures were treated as design variables and optimized by a genetic algorithm to minimize the maximum temperature of the volume. The comparison of obtained trees at different bifurcation levels showed that complexity, i.e. volume-filling, is a common feature of good designs. 
Starting from Murray's law [30, and further inspired by all the above works, researchers have gradually realized that branching and tree-like topologies are a common feature of natural volumeto-point systems. An overwhelming majority of the designs from the intensive study on the volumeto-point problem are hence tree-like, independent of whether the design approaches are targeted at tree structures or not. Remarks like "it can be seen that the design results resemble natural tree networks" can be found in related literature [1, 9, 12, 19, 22, 24, implying that tree structures have been accepted as the optimal topologies of volume-to-point structures. However, the study in this paper shows that lamellar needle-like structures instead of tree structures are the true optimal topologies for volume-to-point structures in the context of steady-state heat conduction for minimum thermal compliance and minimum maximum temperature, respectively. Hence transferring optimization cannot be the pure reason for natural transfer systems being trees. Other physics, load fluctuations, structural requirements, or the need for damage adaption 31, 32 may play an important role in inducing the morphogenesis of branches and even loops.

The intuitive explanation for tree structures not being optimal is simple: The most efficient way to transport heat from a point in the structure to the sink is a straight line. Hence, any detour, as provided by tree structures, is less efficient. Establishing branches may only become an advantage if thicker branches conduct heat nonproportionally to thinner ones. However, this is not the case for the heat conducting structures considered here.

From the applied mathematics community it has long been known that optimal designs for 2D structures under single load conditions can be obtained by so-called rank-1 laminates, both when simple layerings of the high and low conductor. The resulting designs generally have large seemingly gray regions, which denote optimally oriented and proportioned local lamellar microstructures. Visualizing these structures results in infinitely fine, lamellar or needle-like structures. The research presented in this paper was inspired by the apparent discrepancy: Considering that rank-1 lamellar (and hence non-tree) structures have been proven to be optimal by applied mathematicians, why do constructal theory and numerical studies indicate that tree structures are optimal?

The numerical topology optimization approaches reviewed above all make use of design domains made up of a fixed grid where the conductivity of each grid element is a variable. GA-approaches directly solve the discrete material distribution problem, whereas more efficient density or SIMP approaches use continuous design variables and gradients and thereby allow to solve problems 
with finer discretizations. However, to avoid convergence to non-discrete solutions, penalization approaches must be applied to the latter. Penalization makes the optimization problem non-convex and hence prone to getting stuck in local minima (tree structures). In this paper, we show that when good initial guesses are used and hence local minima are avoided, (near-)optimal lamellar designs can also be achieved by penalized density-based approaches. Alternatively, very slow continuation strategies or an additional local volume constraint can be implemented to obtain designs of similar quality, as shown in Appendix A4. In the optimization using these penalized approaches, an implicit minimum length scale may be enforced by the discretization level of the design domain. This may have a strong influence on the topologies of resulting designs [36. However, as our study shows, the best performing designs are still lamellar, even if the minimum length scale is explicitly imposed.

To support our points, the paper is built up as follows. Section 2 contains numerical studies for two simple volume-to-line problems that can be seen as 1D problems and hence allow in-depth analytical verifications and discussions. Section 3 studies the more complex and widely used square volume-to-point problem. The numerical studies are based on three different material interpolation models described in Section 2. These encompass the optimal rank-1 microstructure scheme, the penalized density approach (here realized through the Rational Approximation of Material Properties (RAMP) model), and the simple variable thickness sheet model. Sections 2 and 3 consider the minimum thermal compliance problems, whereas Section 4 considers the same volume-to-line and volume-to-point problems, however for the min-max temperature objective. All numerical and analytical studies support that lamellar structures are superior to tree structures. This observation is further supported by analytical studies and direct comparisons with the literature as provided in Appendices A1-A4. A comparison and discussion of the rank-1 microstructure scheme compared to the variable thickness sheet model is given in Section 5 and overall conclusions are given in Section 6.

\section{Volume-to-line problems}

Instead of studying the volume-to-point problem directly, we start our work from two simpler volume-to-line problems shown in Figures 1 (a) and 2(a). The structures have a spatially uniform 
heat generation rate which is independent of temperature and material distribution 2 . The boundary on the left side, 'line', serves as the heat sink where the temperature is prescribed and the rest of the boundary is heat insulated.

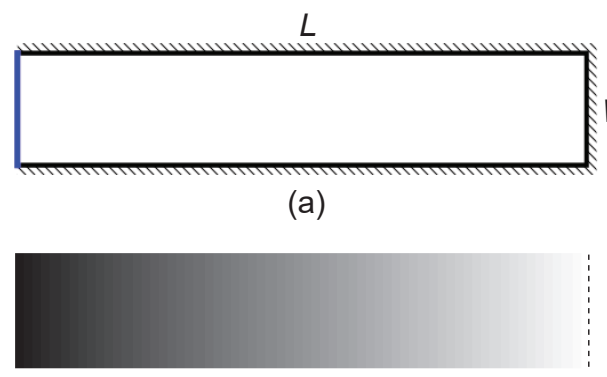

(c)

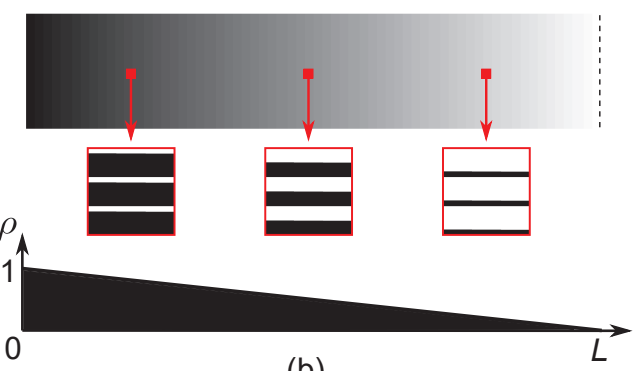

(b)

Figure 1: Rectangular volume-to-line structure. (a) Design domain, (b) Optimal design obtained for the optimal microstructures scheme (grey scale indicating lamellar regions), (c) Optimal design for the variable thickness sheet model - both obtained for the minimum thermal compliance objective.

These two volume-to-line structures are in fact 1D problems due to symmetry. Hence, we can derive the analytical optimal designs in infinite dimensional sense for both the minimum thermal compliance and the minimum-maximum temperature case. In the following, topology optimization of the volume-to-line structures is performed by employing three different material interpolation models to minimize the thermal compliance.

Optimization problem: We consider the standard minimum thermal compliance problem for a structure composed of two materials with high $\left(k_{0}=1\right)$ and low $\left(k_{\min }=0.001\right)$ thermal conductivities, where the volume fraction of the high conductive material is denoted $f$. The uniform heat generation rate is denoted by $g$ and the thermal compliance objective function can hence be written as $\int_{\Omega} g T d \Omega$, where $T$ is the temperature field and $\Omega$ the modelling domain. As seen from the integral, thermal compliance is also equal to the average temperature. In all case element-based design variables are denoted $\rho \in[0,1]$ but have different physcial interpretations as discussed below.

\footnotetext{
${ }^{2}$ Note that some studies in the literature assume design dependent loads, meaning that only low conductivity regions are subject to heat generation. Apart from the heat generation going down with increasing volume fraction, meaning that almost no heat is generated for larger volume fractions, design dependent loads do not seem to have a big influence on the resulting topologies for the minimum thermal compliance problem. However, for the minimum maximum temperature problem, design dependent loads result in weird an un-intended designs, where areas most distant from the sink are fully covered by high conducting material that prevent heat being generated in those areas and hence (artificially) lowering the maximum temperature (see Figure A3.2). For this reason we here study the more natural design-independent problem, however, Appendix A3 considers the design-dependency in order to allow direct comparison with the literature.
} 

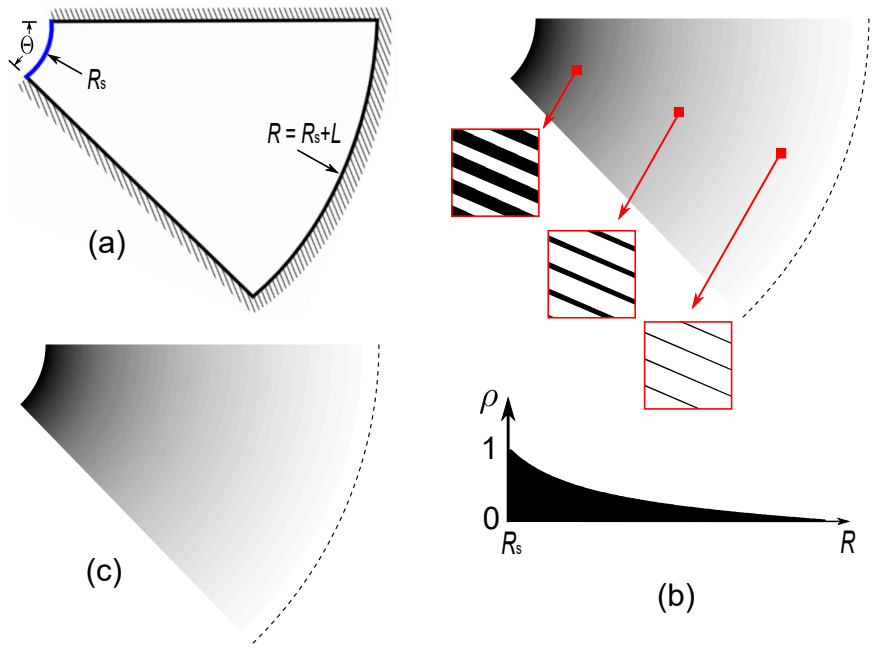

(b)

Figure 2: Circular volume-to-line structure. (a) Design domain (one eigth of the full domain reduced by symmetry considerations), (b) Optimal design obtained for the optimal microstructures scheme (grey scale indicating lamellar regions), (c) Optimal design for the variable thickness sheet model - both obtained for the minimum thermal compliance objective.

A formal definition of the design problem can be found in Appendix A1. The Method of Moving Asymptotes (MMA) algorithm [37] is used to solve the optimization problems.

Optimal microstructures scheme: It is known that rank-1 laminates are optimal for minimum thermal compliance problems and min-max temperature problems under single load conditions [33 35]. The homogenized orthotropic material conductivity of a rank-1 laminate is

$$
\begin{aligned}
& k_{11}(\rho)=\rho k_{0}+(1-\rho) k_{\min }=k_{\min }+\rho\left(k_{0}-k_{\min }\right) \\
& k_{22}(\rho)=\frac{k_{0} k_{\min }}{\rho k_{\min }+(1-\rho) k_{0}} \\
& k_{12}=k_{21}=0
\end{aligned}
$$

where, $k_{11}$ and $k_{22}$ are effective axial conductivities in the material reference system, $\rho \in[0,1]$ is here the relative density of high conductive material. During the optimization process, the laminate orientation is optimized alongside the relative density.

Rational Approximation of Material Properties (RAMP) model[29]: Density-based topology optimization approaches make use of penalized material interpolations schemes to enforce 0-1 solutions despite using continuous variables. Popular schemes include the SIMP and RAMP 
interpolations and here we use the latter as given by

$$
k(\rho)=k_{\min }+\frac{\rho}{1+q(1-\rho)}\left(k_{0}-k_{\min }\right)
$$

where, $q \geq 0$ is the penalization parameter and $\rho \in[0,1]$ is aslo here the relative density of high conductive material. Conclusions of this work do not change if we instead would apply the SIMP interpolation scheme.

Variable thickness sheet model [38]: If we set $q=0$ in (2), we obtain the so-called variable thickness sheet model, where $\rho$ can be interpreted as the out-of-plane element-wise thickness. It does not solve the original problem of distributing two distinct materials but can instead be interpreted as thickness varying material distribution problem. In essence, it is a model in "dimension $2 \frac{1}{2}$ " which cannot be attained by composites of two materials [39]. Nevertheless, we include it here as a reference model and because it reveals additional insight into the design problem. The isotropic material conductivity is given as

$$
k(\rho)=k_{\min }+\rho\left(k_{0}-k_{\min }\right)
$$

It turns out that for a given temperature field and bulk material density, variable thickness sheets have the same energy density as optimal rank-1 laminates since transverse heat conduction is zero. Therefore it can be expected that designs of the optimal microstructures scheme and the variable thickness sheet model should be the same for the minimum thermal compliance objective.

Analytical solution: The analytical derivations of the optimal designs are given in Appendix A1. In order to compare the topology optimization designs with the analytical solutions, the upper bounds for the volume of high conductive material are chosen in such a way that the maximum local material density in the optimal designs is exactly 1 . As derived in the appendix, the resulting upper volume bound is hence $f=0.4995$ for the rectangular structure and about $f=0.209$ for the circular structure.

Numerical considerations: Since the minimum thermal compliance problems based on the optimal microstructures scheme and variable thickness sheet model are (quasi-)convex, the optimization should be able to converge to the global minimum starting from an arbitrary initial guess. In this study, a uniform initial guess is used satisfying the volume constraint. The rectangular structure is partitioned into $200 \times 40$ four-node elements and the circular structure into 
$100 \times 100$ elements. The density filter 40 , 41 is used to avoid checkerboard patterns with filter radius $R_{\min }=\frac{1.5 L}{200}$ for the rectangular structure and $R_{\min }=\frac{1.5\left(R-R_{\mathrm{s}}\right)}{100}$ for the circular one. Periodic boundary condition is applied for the density filter. For the optimal microstructures scheme, the density variables are optimized by the MMA algorithm while the orientations of rank-1 laminates are set to coincide with the directions of heat flux at element centroids. The optimization terminates when the maximum change of design variables between iterations is smaller than 0.001 . Considering that the resulting designs here are composed of microstructures or sheets with different thicknesses, no minimum length scale is imposed.

In the optimization using the RAMP model, an implicit minimum length scale is imposed by design discretizations. It is known from [36] that a coarse mesh, i.e. a large implicit minimum length scale, restricts the design freedom and results in local minima that are far from being optimal. Therefore the robust formulation 42 that can enforce an explicit minimum length scale is used to study the effect of minimum length scale and to ensure convergence to discrete designs. However, with penalization, the minimum thermal compliance problem becomes non-convex and the optimization is prone to getting stuck into local minima. Small variations in optimization parameters can lead to dramatic changes in design results. Hence, it makes sense to try different initial guesses. Qualified initial (lamellar) guesses are constructed by using the following equation:

$$
\rho(\varepsilon, \eta)=\min \left\{2 \rho_{0}(\varepsilon)\left[\frac{1}{2}-\frac{1}{2} \cos (P \cdot 2 \pi \eta)\right], 1\right\}
$$

where $\varepsilon=x / L$ and $\eta=y / W$ for the rectangular structure, $\varepsilon=r /\left(R-R_{\mathrm{s}}\right)$ and $\eta=\theta / \Theta$ for the circular structure, $\rho_{0}$ is the analytical solutions and $P$ controls the period of initial guesses. Resulting initial guesses are seen in the top row of Figure 3 and the leftmost column of Figure 4

The optimization using the qualified initial guesses is carried out for structures with different aspect ratios, $\Gamma=\frac{L}{W}=\frac{R-R_{\mathrm{s}}}{R_{\mathrm{s}} \Theta}=\frac{5}{2^{0}}, \frac{5}{2^{-1}}, \ldots, \frac{5}{2^{-5}}$. The largest rectangular structure is partitioned into $2400 \times 480$ elements and the largest circular one into $1200 \times 1200$ elements. Three minimum length scales, $b=0.005 \mathrm{~L}, \frac{2}{3} \times 0.005 \mathrm{~L}, \frac{1}{3} \times 0.005 \mathrm{~L}$, are imposed respectively in both phases. The filter radius of the density filter is $R_{\min }=1.1 b$ and periodic boundary conditions are applied. A small penalty parameter $q=2$ is used. The projection thresholds in the robust formulation are $\eta_{\mathrm{e}}=0.7066, \eta_{\mathrm{i}}=0.5, \eta_{\mathrm{d}}=0.2934$ computed following the method in [43]. The parameter $\beta$, controlling the sharpness of projection, is doubled every 50 iterations from 1 to 64 . The volume 
constraint is imposed on the dilated design and the upper bound on volume is reevaluated every 20 iterations. The optimization terminates when the maximum design variable change between iterations is smaller than 0.01 or the number of iterations reaches 1000. After convergence, the design results are projected to strict black-white designs, while preserving the volume of the high conductive material.

Numerical results: Design results for the optimal microstructures scheme and variable thickness sheet model are shown in Figure 1 (b), (c) and Figure 2 (b), (c). In these two material models, intermediate material densities also work well in comparison to material cost, so large gray regions can be found. When zooming in on designs of the optimal microstructures scheme and examining the material fractions and orientations of the underlying rank-1 laminates, we observe that the optimized designs are needle structures. This observation matches the analytical solutions and gives us inspiration for the construction of initial guesses Eq. (4) for the optimization by the RAMP model. For thermal compliance minimization problems under single load conditions, the optimal structures have a constant thermal energy density distribution in regions with intermediate densities. Therefore the density of high conductive material changes linearly with respect to the distance away from the heat sink for the optimal rectangular structure, and changes nonlinearly for the optimal circular structure. Straight linear and non-linear decaying needles can be seen in the optimal designs of the rectangular and circular structures, respectively.

Figures 3 and 4 give the designs of the RAMP model, which clearly show that for this optimization approach, even the simple 1D volume-to-line problems have many local minima. Best designs obtained for each imposed minimum length scale are framed by red boxes, clearly showing that lamellar structures always are superior to tree structures. By checking the temperature gradients of initial guesses and design results, we observe that if a high conductive local region exists (e.g., a branch), the heat conduction in its neighborhood will point toward this high conductive local region instead of towards the heat sink. This explains why the optimization converges to local minima as soon as a branch has appeared and why most local minima are tree structures with branches. According to the summarized results in Figure 5 designs with the smallest minimum length scale perform better than others from the same initial guess. This can be explained by the topologies of designs. Longer and thinner high conductive branches or needles arise with the decrease of the minimum length scale. Hence more regions have direct access to high conductive paths and the structure is cooled more evenly. 


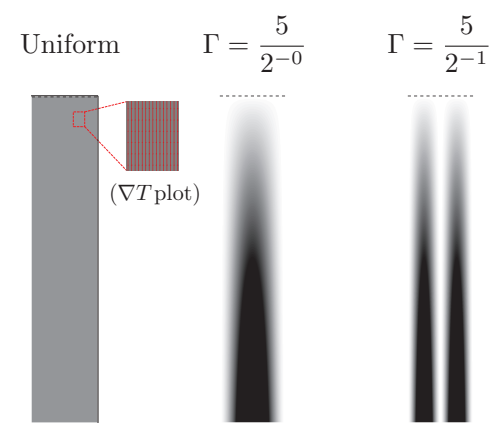

$\Gamma=\frac{5}{2^{-2}}$

$\Gamma=\frac{5}{2^{-3}}$

$\Gamma=\frac{5}{2^{-4}}$

$\Gamma=\frac{5}{2^{-5}}$
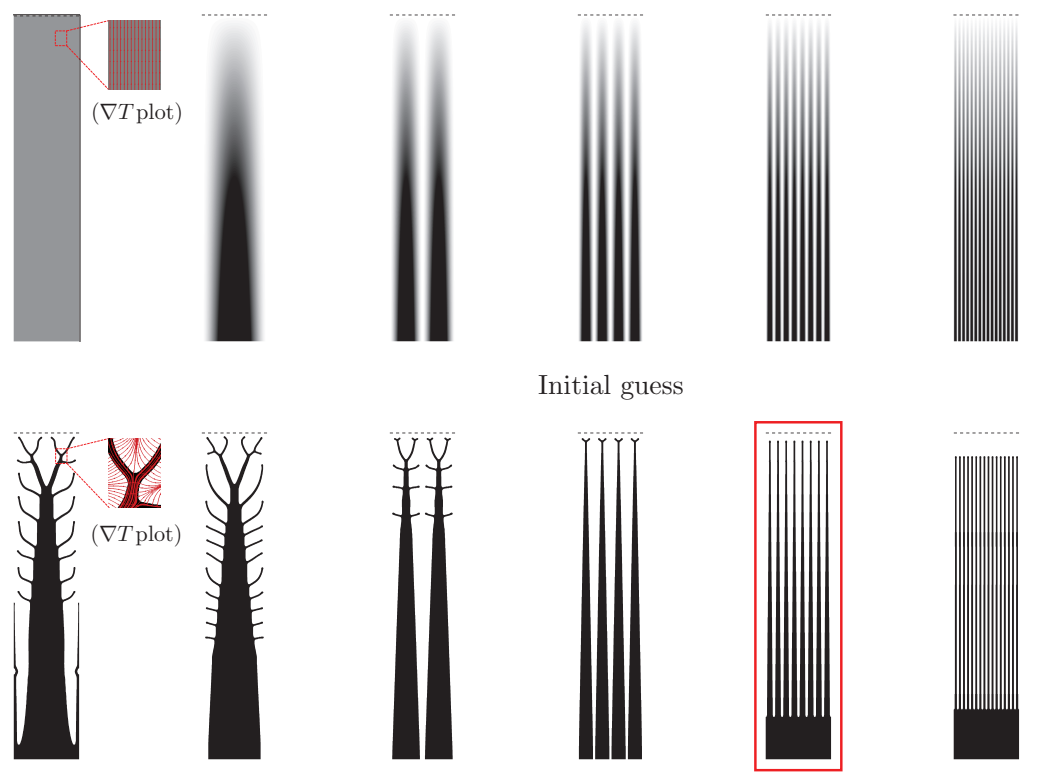

Initial guess
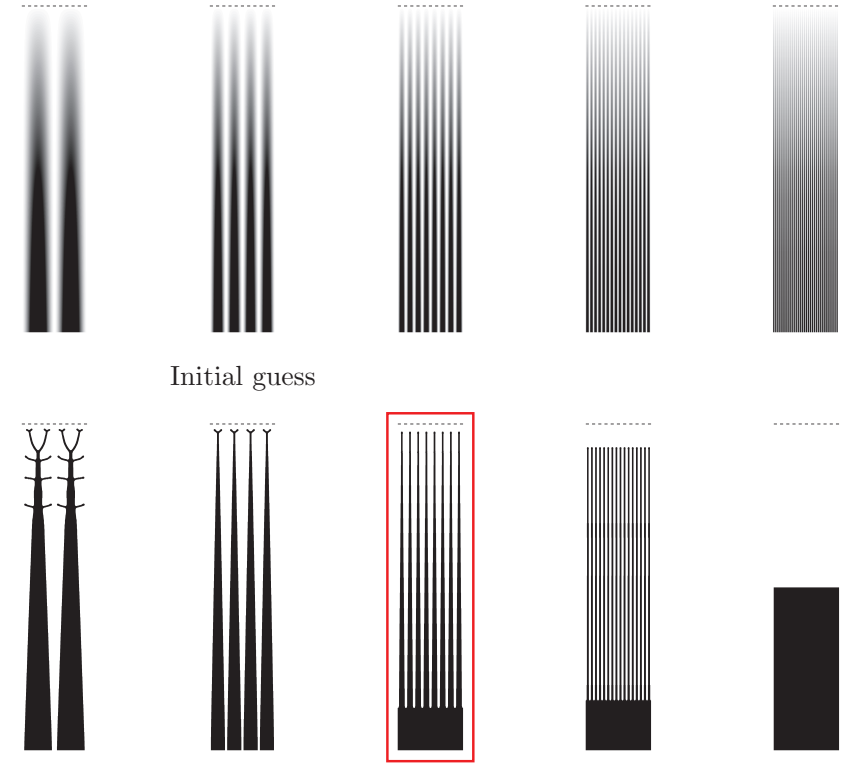

Minimum length scale $b=0.005 L$
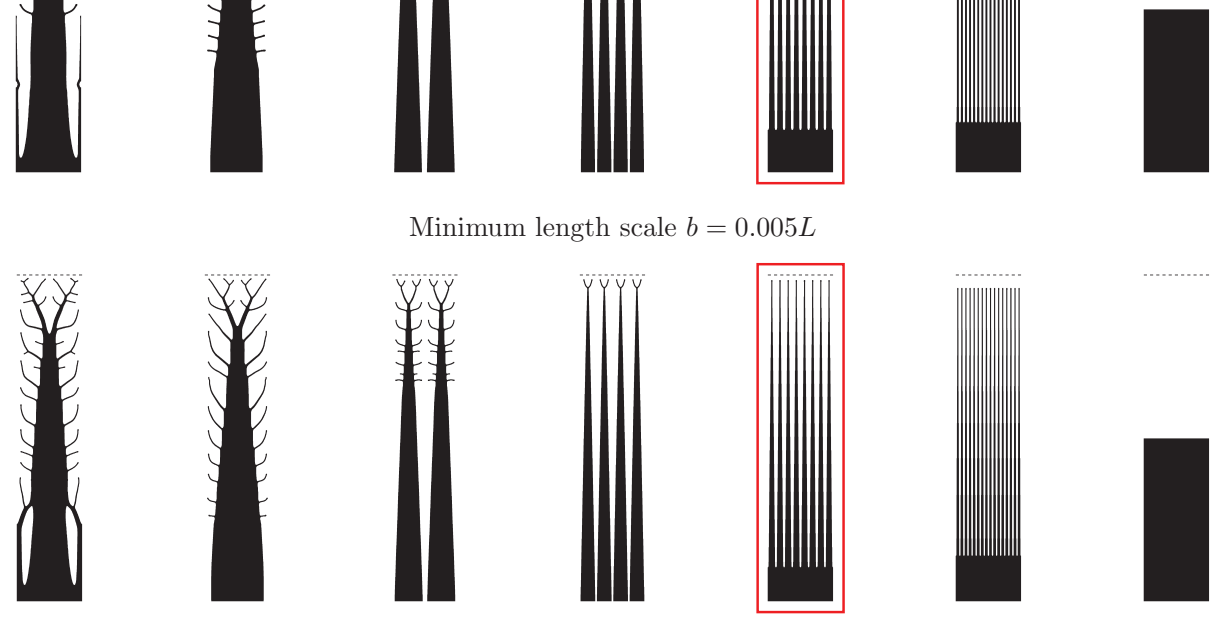

Minimum length scale $b=\frac{2}{3} \times 0.005 L$
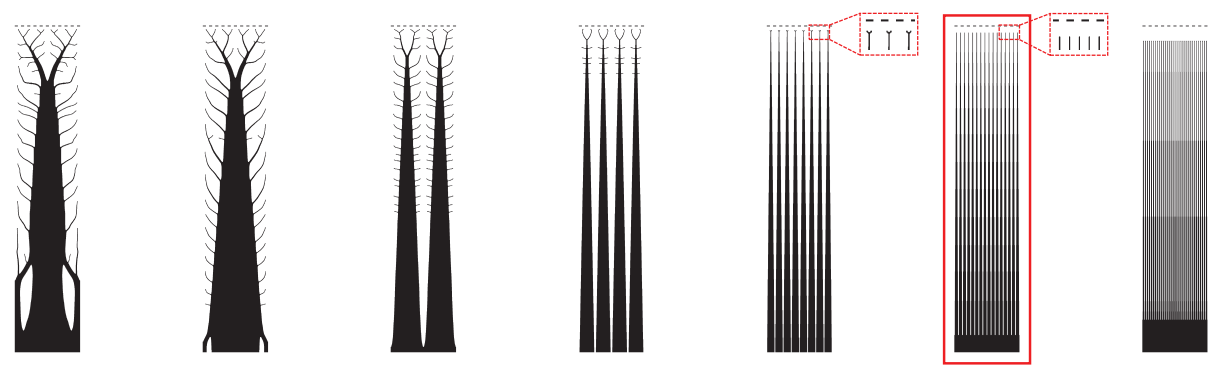

Minimum length scale $b=\frac{1}{3} \times 0.005 \mathrm{~L}$

Figure 3: Designs of the rectangular volume-to-line structure with different aspect ratios of full design domain for minimum thermal compliance. Red boxes indicate designs with the best objective function values for the prescribed length scale. 


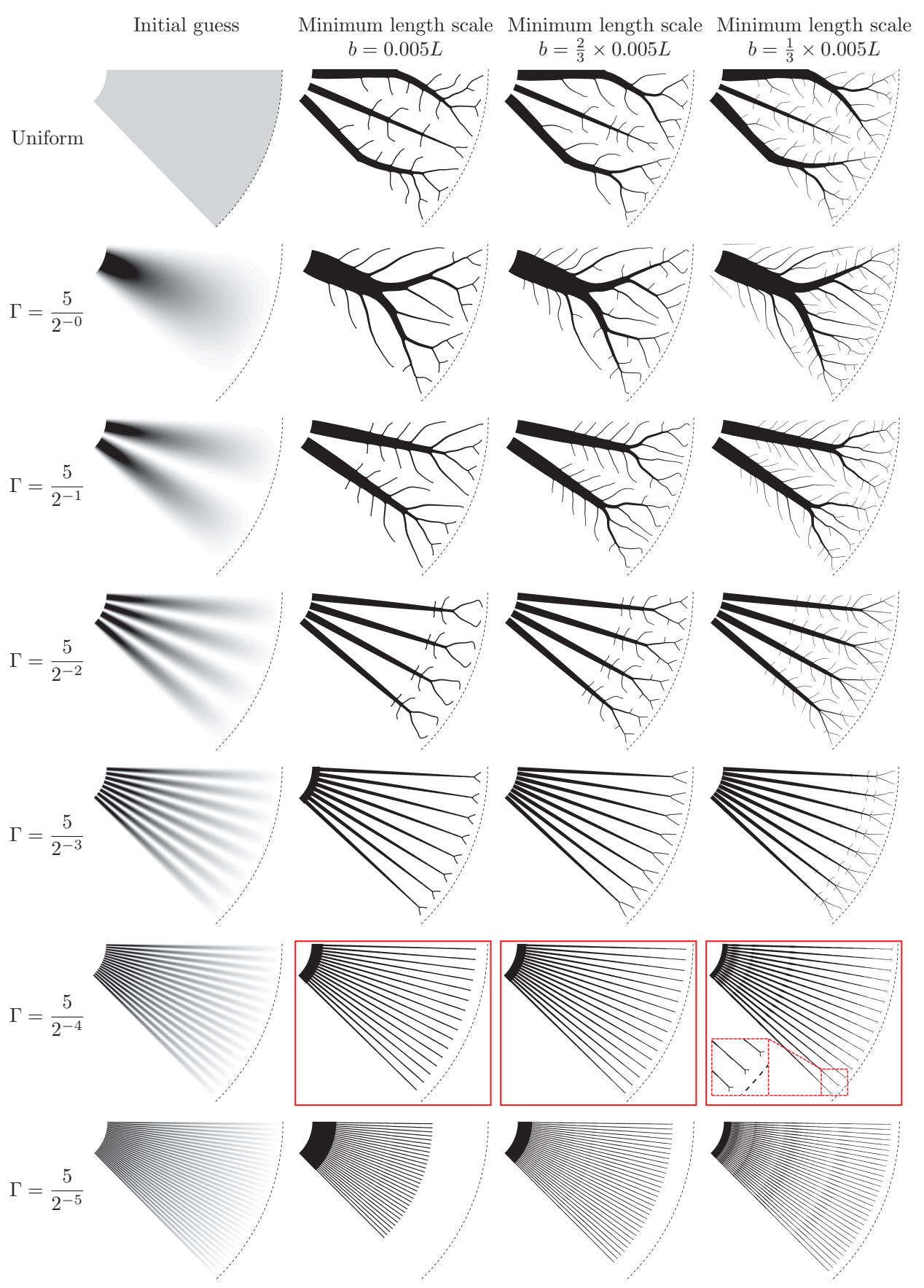

Figure 4: Designs of the circular volume-to-line structure with different aspect ratios of full design domain for minimum thermal compliance. Red boxes indicate designs with the best objective function values for the prescribed length scale. 


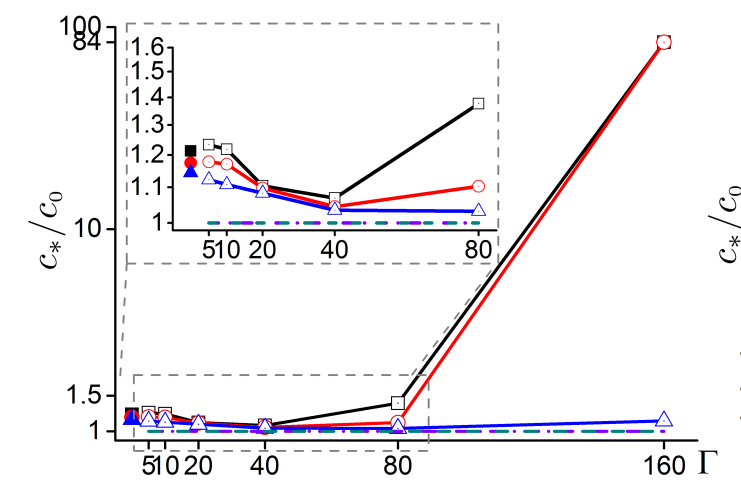

(a)

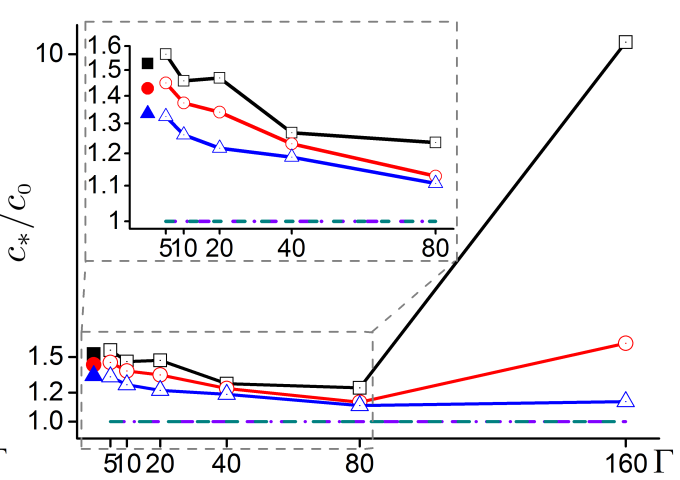

(b)

- Unifrom initial guess, $b=0.005 \mathrm{~L} \quad-\square$ Needles initial guess, $b=0.005 \mathrm{~L}$

- Uniform initial guess, $b=2 \times 0.005 \mathrm{~L} / 3-$ Needles initial guess, $b=2 \times 0.005 \mathrm{~L} / 3 \quad \cdots$ Variable thickness sheet model

$\Delta$ Uniform initial guess, $b=0.005 \mathrm{~L} / 3 \quad-$ Needles initial guess, $b=0.005 \mathrm{~L} / 3$

Figure 5: Objective function ratios of optimized designs $\left(c_{*}\right)$ compared to the corresponding analytical solutions $\left(c_{0}\right)$ for minimum thermal compliance from Figures 3 and 4 (a) Rectangular volume-to-line structure, (b) Circular volume-to-line structure

By comparing the designs subject to the same minimum length scale but originating from

different initial guesses, we find that conducting heat directly to the heat sink (needle structures) is better than collecting heat by small branches at first and then conducting to the heat sink by main trunks (tree structures). Filling the whole structure is also important for a uniform cooling. Although the local minima obtained by the RAMP model have varying performance, the optimization can still converge to near-optimal designs when good initial guesses are used. Here the good initial guesses consist of a certain number of gray needles close to the (near-)optimal minima. The obtained near-optimal designs are lamellar needle structures, in agreement with the analytical solutions and the designs from the two other material models. Also interesting is that this conclusion still holds when minimum length scale is imposed. As for the minimum length scale $b=\frac{1}{3} \times 0.005 L$ for the circular structure, a better design consisting of 24 needles with no branches is obtained by using an aspect ratio $\Gamma=120$. The curves in Figure 5 also show that the designs of the circular structure converge slower than the rectangular structure with respect to the number of needles. Hence more needles are needed in the (near-)optimal circular structure. Finally, we note that the quantitative improvement in compliance value for needle structures compared to non-optimal tree structures subject to the same length-scale constraint is in the range of $10-20 \%$ as can be observed in Figure 5 . 

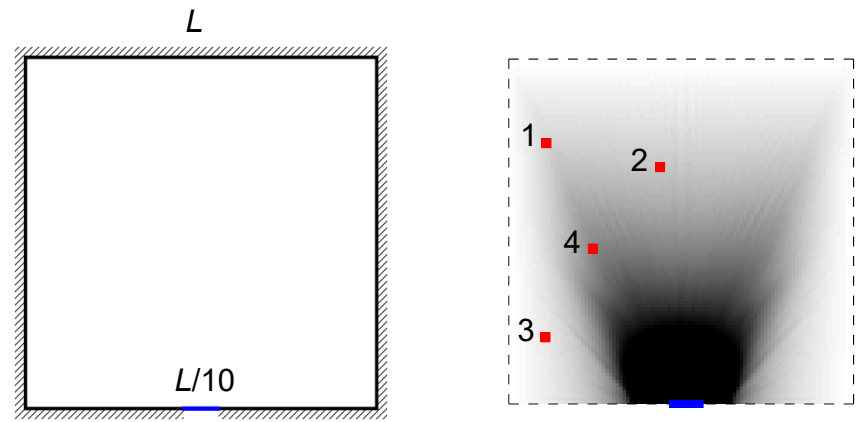

(a)

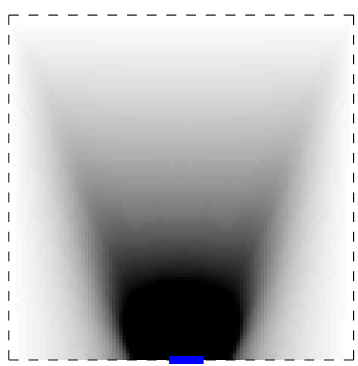

(c)
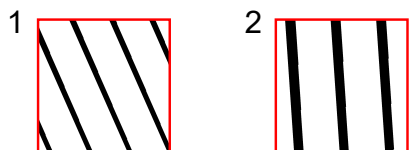

3

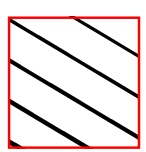

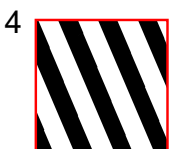

(b)

Figure 6: Volume-to-point structure. (a) Design domain, (b) Optimal design obtained by the optimal microstructures scheme where points 1-4 are enlarged to reveal the underlying optimal microstructure and its orientation, (c) Optimal design for the variable thickness sheet model for minimum thermal compliance.

\section{Volume-to-point problem}

The volume-to-point problem shown in Figure6(a) is studied in this section. The structure has a uniform heat generation rate, the center of the boundary at the bottom is the heat sink and the rest of the boundary is heat insulated. The material interpolation models mentioned in Section 2 are used to minimize the thermal compliance. The upper bound for the volume fraction of high conductive material is $f=0.3$. The structure is discretized into $100 \times 100$ elements in the optimization using the optimal microstructures scheme and variable thickness sheet model and $800 \times 800$ elements for the RAMP model. Three minimum length scales, $b=5 \times 0.005 L, 2 \times 0.005 L, 0.005 L$, are enforced respectively. The implementation details and the values of other parameters are the same as the previous volume-to-line problems.

A uniform initial guess is used for the optimal microstructures scheme and variable thickness sheet model. As for the RAMP model, it is necessary to try different initial guesses due to the existence of many local minima. With the method developed in [44, we construct the initial 
guesses by projecting the design of the optimal microstructures scheme into different macroscopic discrete structures through scaling of the periodicity of the cosine waves. These structures are then smoothed while keeping the topologies unchanged. The qualified initial guesses are shown in the left column of Figure 7 .

The optimal design for the optimal microstructures scheme consists of lamellar rank-1 with spatially varying material fractions and orientations as shown in Figure 6 (b). One way that enables us to interpret the design is to project it into discrete structures [44]. Since the initial guesses in Figure 7 are gray versions of the projected structures with the same topologies, we can see that the projected designs are needle structures subject to an implicit minimum length scale enforced by the discretization. The projected structure corresponding to the initial guess No.1 has a thermal compliance of about 1.1 times that of the optimal rank-1 laminate design. It can be expected that even better projected needle structures can be obtained by further parameter tuning.

Solutions for the optimization using the RAMP model with robust formulation can be found in Figure 7. Clearly, a uniform material distribution is not a good initial guess for the volume-to-point problem. Designs subject to a smaller minimum length scale perform better than others of the same initial guess according to the curves plotted in Figure 8. A conclusion that is quite different from the intuitive and widespread hypothesis obtained from the observation of natural transferring systems and designs in numerous related studies can be made by comparing the designs subject to the same minimum length scale. The best designs obtained for each column (i.e. minimum length scale) are framed by red and again clearly show that needle structures instead of tree structures provide the (near-)optimal topologies for volume-to-point problems and branching is not a preferable way to promote heat conduction when the performance metric is thermal compliance. This conclusion still holds even if a large minimum length scale is imposed.

\section{Min-max temperature problems}

In this section, we study the same volume-to-line and volume-to-point structures as before but now with the aim of minimizing the maximum temperature. Specifically, the maximum temperature objective is approximated by the $p$-norm of nodal temperatures where $p=10$ is used for computational efficiency. The effect of $p$ values on the optimization is studied in Appendix A2, which also confirms that the temperature at the outer rim becomes constant as the $p$ value approaches infinity. Optimizations employing the three material interpolation models mentioned in Section 2 


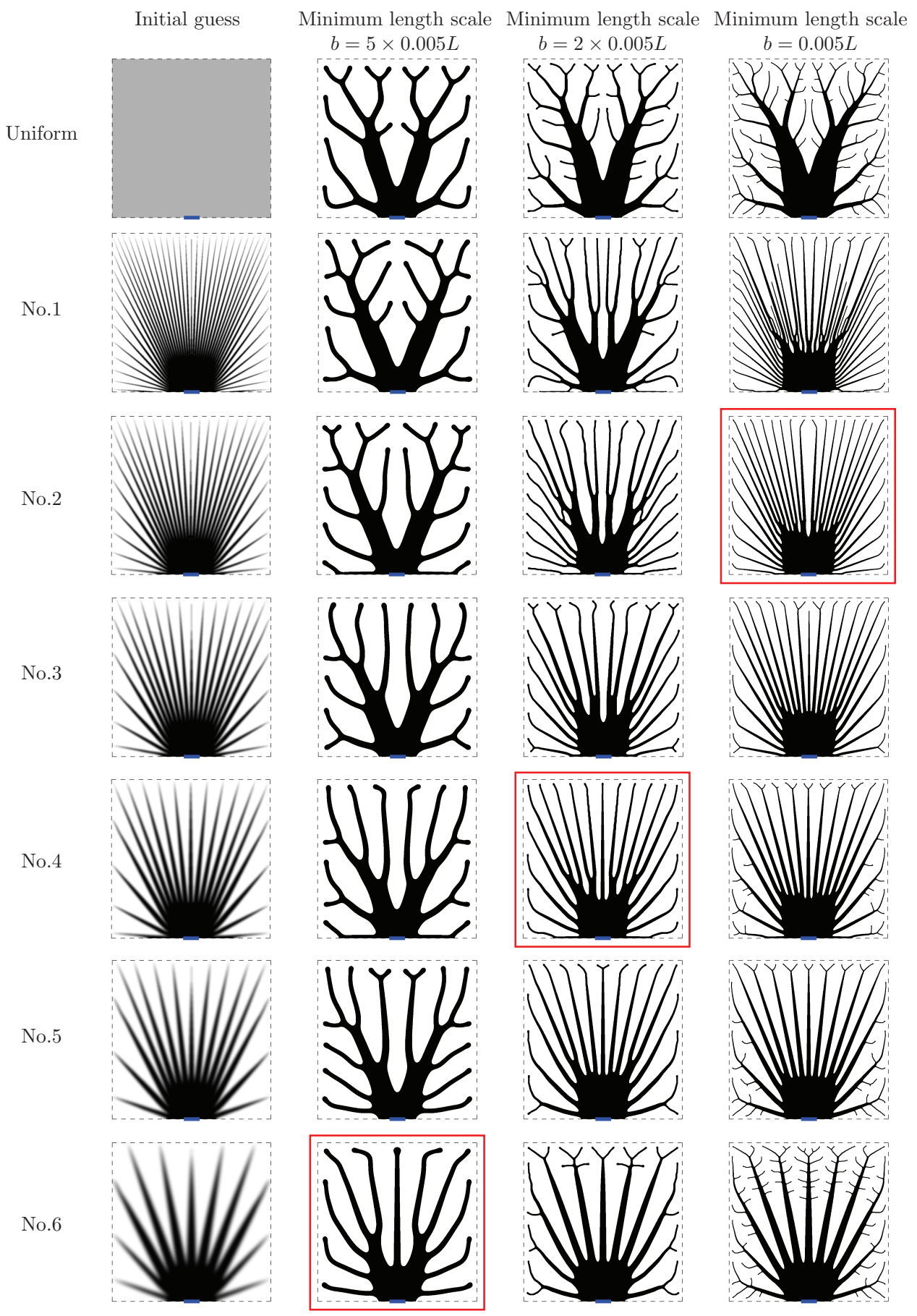

Figure 7: Optimized designs for the volume-to-point structure for minimum thermal compliance obtained using the RAMP scheme. Left column shows starting guesses used for different imposed length-scales. Red boxes indicate designs with the best objective function values for the prescribed length scale 


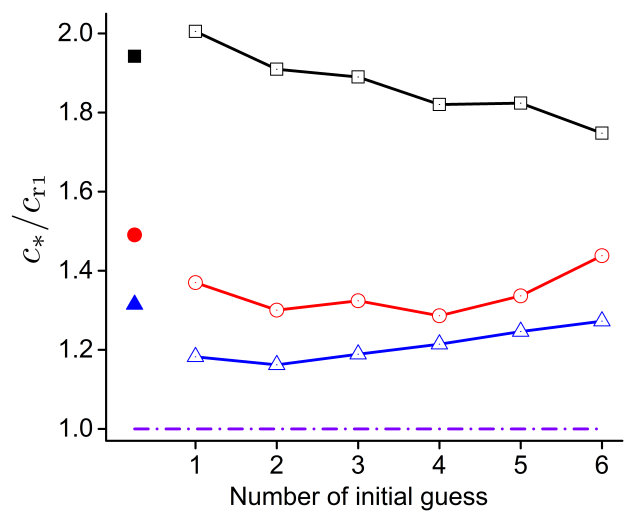

- Uniform initial guess, $b=5 \times 0.005 \mathrm{~L} \quad \square-$ Needles initial guess, $b=5 \times 0.005 \mathrm{~L}$

- Uniform initial guess, $b=2 \times 0.005 \mathrm{~L} \quad-$-Needles initial guess, $b=2 \times 0.005 \mathrm{~L}$

$\Delta$ Uniform initial guess, $b=0.005 \mathrm{~L} \quad \sim$ Needles initial guess, $b=0.005 \mathrm{~L}$

-.-Variable thickness sheet model

Figure 8: Objective function ratios of optimized designs $\left(c_{*}\right)$ compared to the corresponding rank-1 laminates design $\left(c_{\mathrm{r} 1}\right)$ of the volume-to-point structure for minimum thermal compliance

are performed. Design discretizations and parameter settings are the same as for the minimum thermal compliance problems unless otherwise stated. In the optimization based on the optimal microstructures scheme, orientation variables are now also optimized by MMA, different from the minimum thermal compliance problems where directions of heat flux are used. Comparing with the minimum thermal compliance problems, an adjoint problem is required in the optimization, since min-max temperature problems are non-self adjoint (see more details in Appendix A1).

Volume-to-line structures: The analytical optimal designs of volume-to-line structures are derived in Appendix A1. The volume fraction of the high conductive material is about $f=0.6663$ for the rectangular structure and about $f=0.2488$ for the circular structure when the maximum local density in the optimal designs is exactly 1. Design results for the optimal microstructures scheme and the variable thickness sheet model are shown in Figure 9. The optimized designs for rank-1 laminates both consist of straight and nonlinearly decaying needles when no minimum length scale is enforced. Similar designs can also be obtained by the variable thickness sheet model for these 1D problems where no transverse heat transfer is present for the optimal structures.

By again utilizing Eq. (4), initial guesses for the optimization of the RAMP model and robust formulation are constructed. A series of optimization are performed and strict black-white designs are obtained. The ratios of objective function and maximum temperature of optimized designs to analytical solutions are plotted in Figures 10 and 11 together with designs with the best objective 


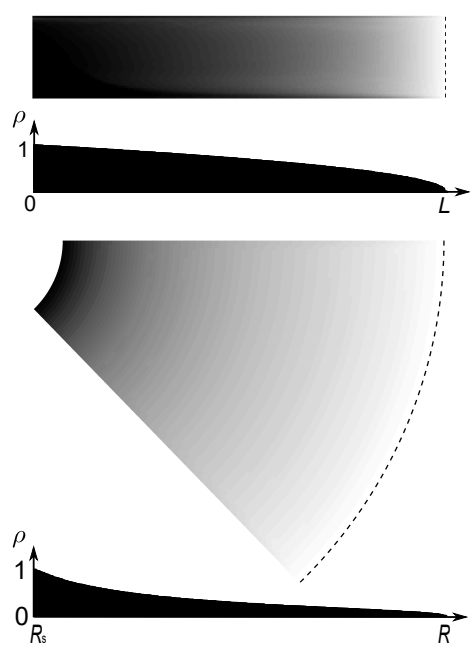

(a)
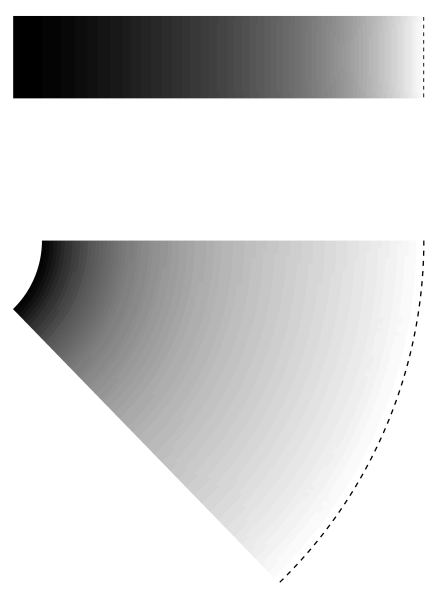

(b)

Figure 9: Optimal designs for volume-to-line structures by (a) the optimal microstructures scheme with grey scale indicating lamellar microstructure, (b) the variable thickness sheet result for min-max temperature.

function values. Apparently, the best designs for all prescribed minimum length scales are again lamellar needles.

Volume-to-point structure: Because of the non-linearity of min-max temperature problems, even the optimization by rank-1 laminates converges to inferior local minima when a uniform initial guess is used. Thus the minimum thermal compliance design by rank-1 laminates is used as the initial guess for the optimization by rank-1 laminates and variable thickness sheet models for the min-max temperature problem. The value of $p$ in $p$-norm is doubled from 1 to 10 every 50 iterations to stabilize the optimization process. As shown in Figure 12, designs for rank-1 laminates and the variable thickness sheet model now have different topologies and the latter has a worse performance. This is because, as will be explained in Section 5 and in Appendix A1, min-max temperature problems are non-self adjoint and the gradients of temperature and adjoint solution are in general not (anti-)parallel to each other.

The optimal design obtained for rank-1 laminates is projected to construct qualified initial guesses for the optimization by the RAMP model. The performance of design results and the best designs together with corresponding initial guesses are shown in Figure 13 Obviously, the best designs are still needles. In addition, the projected structures of the minimum thermal compliance 


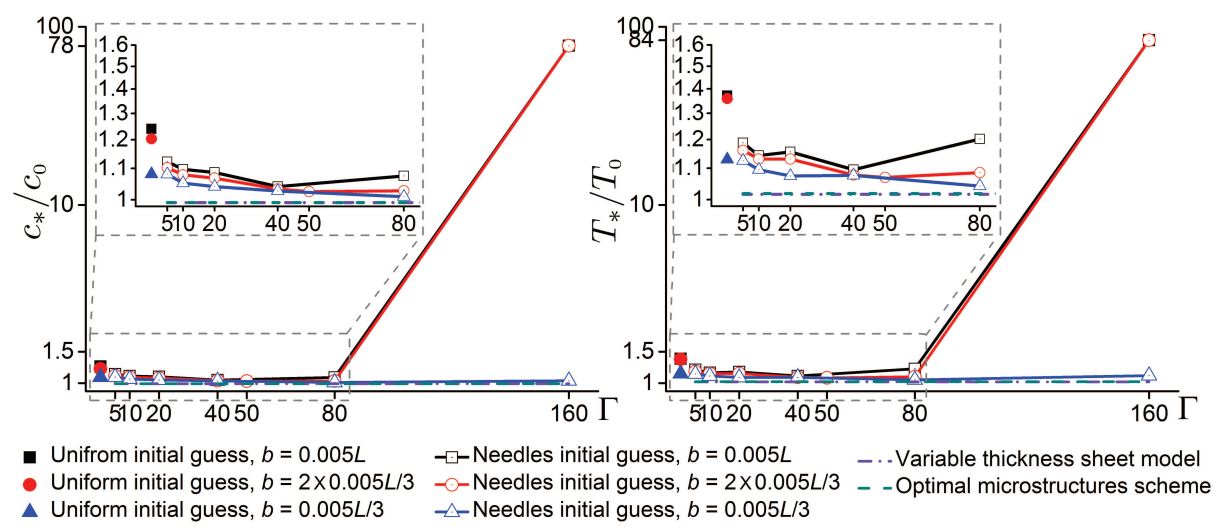

(a)

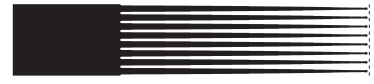

$\Gamma=40, b=0.005 L$
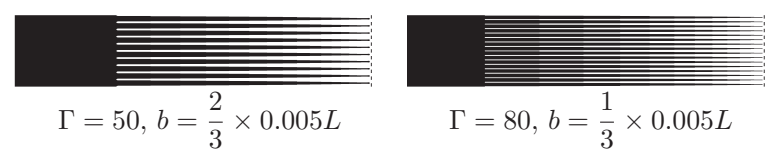

(b)

Figure 10: Designs of the rectangular volume-to-line structure for min-max temperature. (a) Ratios of objective function and maximum temperature, (b) Designs with the best objective function values and maximum temperature for the prescribed length scale.

design have straight and linearly decaying needles at the sides of the design domain, whereas nonlinear decaying needles are observed at these regions for the min-max temperature problem. This is also because the gradients of the direct and adjoint solutions for the min-max temperature problem have quite different directions in these regions.

The results presented in this section were obtained for a $p$-norm value of 10 . Obviously, the true min-max objective is only obtained for $p$ approaching infinity. However, high $p$-norm values make the problem extremely non-linear and hence difficult to solve numerically. To investigate the effect of higher $p$-norm values, Appendix A2 studies the results for $p$-norm values varying from 1 to 60 . For $p=1$, the result is very similar to the minimum thermal compliance result whereas for $p=60$, the boundary temperature is almost constant as would be expected. The designs obtained for $p=10$ and $p=60$ are virtually indistinguishable and the maximum temperature obtained for the $p=10$ case is only a few percent higher than for the $p=60$ case, hence supporting the use of $p=10$ as a good compromise between numerical efficiency and accuracy. 

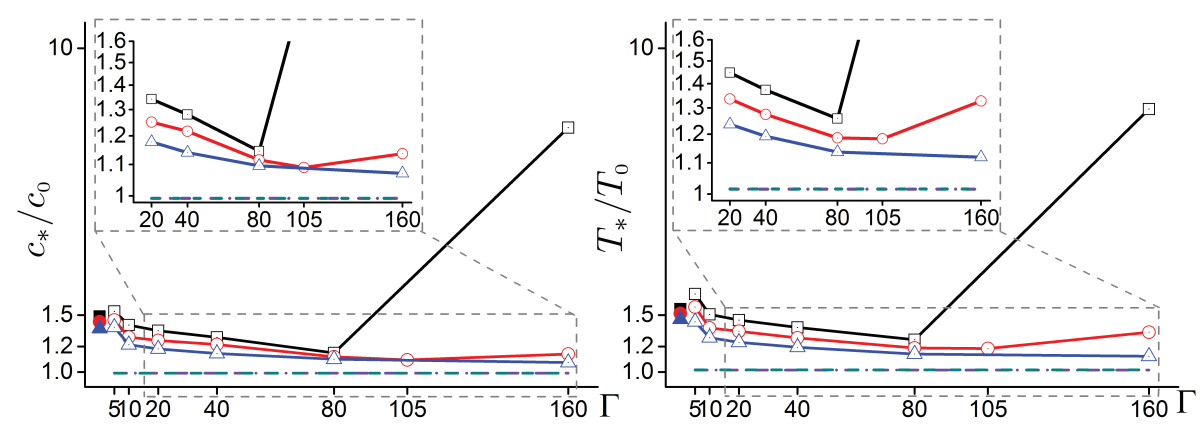

- Unifrom initial guess, $b=0.005 \mathrm{~L}$

-..Variable thickness sheet model

- Uniform initial guess, $b=2 \times 0.005 \mathrm{~L} / 3 \quad-$-Needles initial guess, $b=2 \times 0.005 \mathrm{~L} / 3$ - Optimal microstructures scheme

Uniform initial guess, $b=0.005 \mathrm{~L} / 3$ $\triangle-$ Needles initial guess, $b=0.005 \mathrm{~L} / 3$

(a)

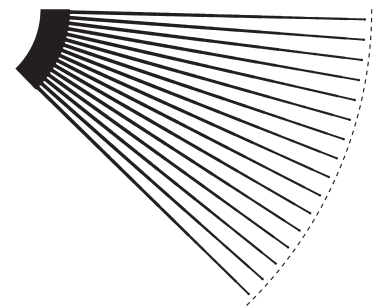

$\Gamma=80, b=0.005 L$

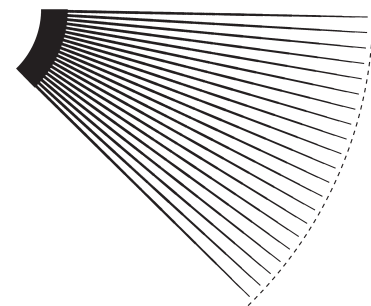

$\Gamma=105, b=\frac{2}{3} \times 0.005 L$

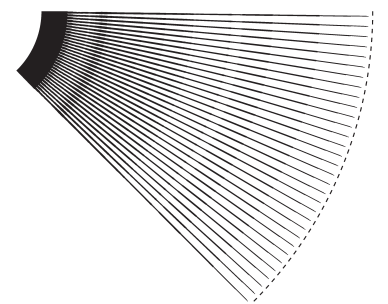

$\Gamma=160, b=\frac{1}{3} \times 0.005 L$

(b)

Figure 11: Designs of the circular volume-to-line structure for min-max temperature. (a) Ratios of objective function and maximum temperature, (b) Designs with the best objective function values and maximum temperature for the prescribed length scale

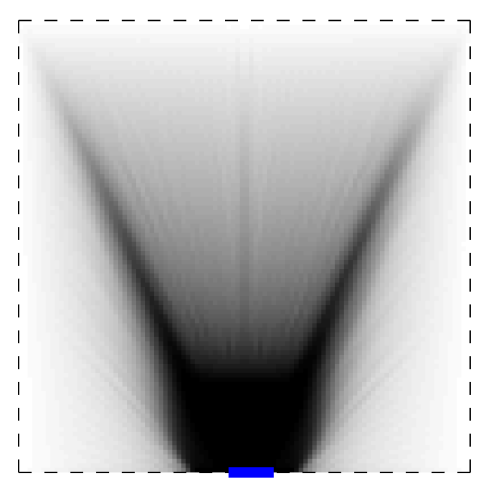

(a)

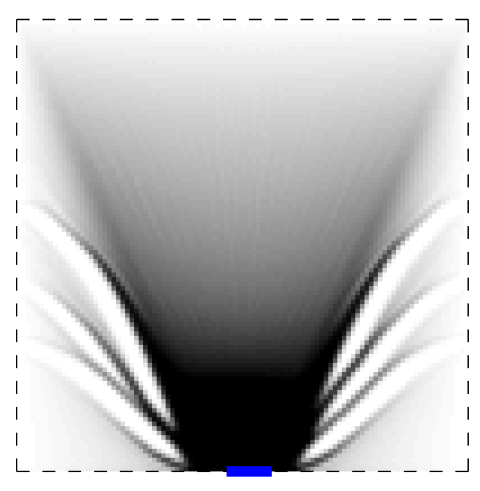

(b)

Figure 12: Optimized designs for the volume-to-point structure by (a) the optimal microstructures scheme, (b) the variable thickness sheet model for min-max temperature objective. 

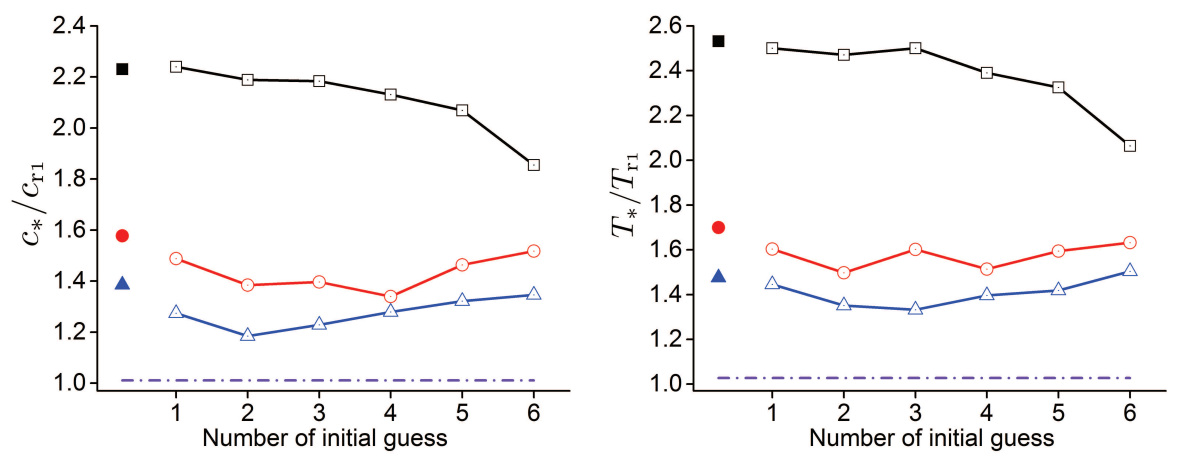

- Unifrom initial guess, $b=5 \times 0.005 \mathrm{~L}-\square$ Needles initial guess, $b=5 \times 0.005 \mathrm{~L} \ldots$-..Variable thickness sheet model

- Uniform initial guess, $b=2 \times 0.005 \mathrm{~L}-\infty$-Needles initial guess, $b=2 \times 0.005 \mathrm{~L}$

$\Delta$ Uniform initial guess, $b=0.005 \mathrm{~L} \quad \triangle$ Needles initial guess, $b=0.005 \mathrm{~L}$

(a)

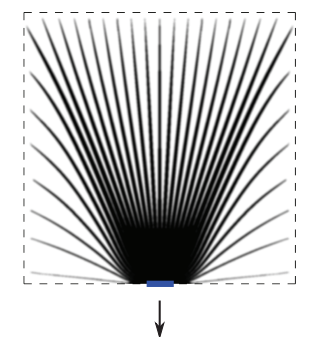

$\downarrow$

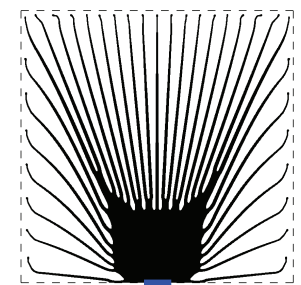

Initial guess $2, b=0.005 L$

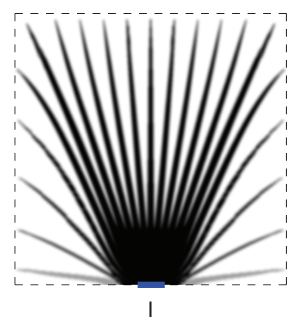

$\downarrow$

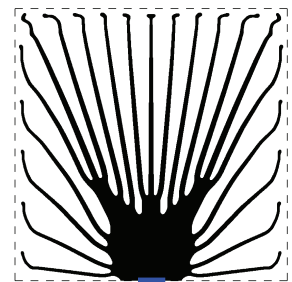

Initial guess $4, b=2 \times 0.005 L \quad$ Initial guess $6, b=5 \times 0.005 L$
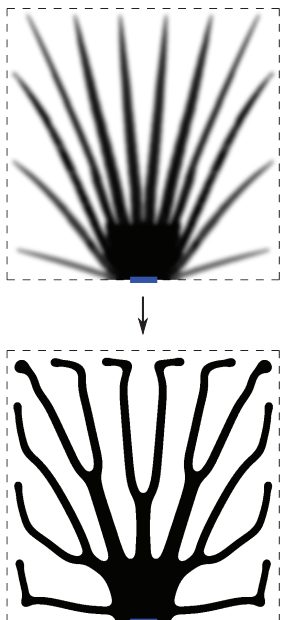

(b)

Figure 13: Designs of the volume-to-point structure for min-max temperature. (a) Ratios of objective function and maximum temperature, (b) Designs with the best objective function values for the prescribed length scale 


\section{Optimal rank-1 model vs. the variable thickness sheet model}

This section is devoted to a discussion of the similarities and differences between the rank- 1 and variable thickness sheet models used in the minimum thermal compliance problems and min-max temperature problems, respectively.

Based on the derivations in 33, 35 and in Appendix A1, the considered topology optimization problems can be reduced to the following local problem.

$$
\min _{\mathbf{D}} \nabla \mu \cdot \mathbf{D} \nabla T \geq\|\nabla \mu\|\|\nabla T\|\left(\lambda_{\min } \cos ^{2} \theta-\lambda_{\max } \sin ^{2} \theta\right)
$$

'Local' means we are considering a neighborhood of a point in the design domain and the material volume fraction $\rho$, temperature field $T$ and the adjoint solution $\mu$ averaged over the neighborhood by the homogenization approach [45] are fixed. In this case, the topology optimization problem consists in finding the local composite that minimizes $\nabla \mu \cdot \mathbf{D} \nabla T$, where $\mathbf{D}=\lambda_{1} \mathbf{e}_{1} \mathbf{e}_{1}^{\mathrm{T}}+\lambda_{2} \mathbf{e}_{2} \mathbf{e}_{2}^{\mathrm{T}}$ is the material conductivity tensor with $\lambda_{i}, \mathbf{e}_{i}(i=1,2)$ denoting eigenvalues and eigenvectors, respectively.

The lower bound of the reduced local objective function $\nabla \mu \cdot \mathbf{D} \nabla T$ in Eq. (5) can be realized by rank- 1 laminates if the normal to the material layers bisects the angle, $2 \theta$, enclosed by $\nabla T$ and $\nabla \mu$ and $\lambda_{\min }=\frac{k_{0} k_{\min }}{\rho k_{\min }+(1-\rho) k_{0}} \leq \lambda_{1}, \lambda_{2} \leq \lambda_{\max }=\rho k_{0}+(1-\rho) k_{\min }$. Because minimum thermal compliance problems are self-adjoint with $\mu=-T$, material layers of the optimal rank-1 laminates orient parallel to $\nabla T$ and $\theta=\frac{\pi}{2}$. As a result, the above lower bound can also be achieved by the variable thickness sheet model with the same material density for the minimum thermal compliance case. In contrast, the min-max temperature problem is non-self adjoint. Combining this with the fact that the variable thickness sheet model dictates an isotropic material with eigenvalues both equal to $\lambda_{\max }$, explains why the design for the variable thickness sheet model for the min-max temperature objective is inferior to the design for rank-1 laminates.

In conclusion, the variable thickness sheet model can be used as a simple substitute for the rank-1 model for the minimum thermal compliance problem. The resulting grey scale structure can subsequently be interpreted as a locally lamellar microstructure with proportion of the high conduction material equal to the local thickness variable and oriented in the direction of the temperature gradient. In contrast, the variable thickness sheet model cannot provide a simplified model for the 
material needed for minimization of the maximum temperature.

\section{Discussions and conclusions} meaningful design-dependent problems. However, even for this problem, lamellar needle structures are superior to all results from the literature with largest gains obtained for higher conductivity 
contrasts.

For minimum thermal compliance and min-max temperature problems under single load conditions, optimal designs can be realized by rank-1 laminates. That is, rank-1 laminates provide the optimal local composites. For compliance problems, the variable thickness sheet model may substitute the more complex rank-1 laminates model and result in equal designs. However, for the non-self adjoint min-max temperature problem, anisotropic thermal conductivities are required and hence the variable thickness sheet model becomes non-optimal.

\section{Acknowledgments}

The authors thank Jeroen P. Groen for his help with projecting rank-1 designs of the volumeto-point structures to the macro scale. The authors also thank Robert V. Kohn and Andrej V. Cherkaev for valuable discussions on the optimality of rank-1 laminates and the variable thickness sheet model for (non-)self adjoint heat conduction problems. The first author thanks the China Scholarship Council (CSC) for the financial support to this work. The third author acknowledges support from the TopTen project sponsored by the Danish Council for Independent Research (DFF4005-00320).

\section{Appendix A1. Analytical solutions}

\section{A1.1 Optimization problem}

When allowing composites of high and low conductive materials in optimized designs, the optimization problems studied in this paper can be formulated as

$$
\begin{array}{cl}
\min _{\rho} & c(T, \rho) \\
\text { s.t. } & \nabla \cdot(\mathbf{D}(\rho) \nabla T)+g=0 \text { in } \Omega \\
& \int_{\Omega} \rho d \Omega \leq f \int_{\Omega} d \Omega \\
& 0 \leq \rho \leq 1
\end{array}
$$

where, $\rho$ represents the material density distribution, $T \in T_{\text {ad }}$ is the temperature field, $c(T, \rho)=$ $\int_{\Omega} F(T, \rho) d \Omega$ is the objective function, $\mathbf{D}=\lambda_{1} \mathbf{e}_{1} \mathbf{e}_{1}^{\mathrm{T}}+\lambda_{2} \mathbf{e}_{2} \mathbf{e}_{2}^{\mathrm{T}}$ is the effective material conductivity tensor, $g$ is the heat generation rate which is independent on material distribution, $f$ is the allowable 
volume fraction of high conductive material and $T_{\mathrm{ad}}$ is the set of admissible temperature fields satisfying the following boundary conditions.

$$
T=0 \text { on } \partial \Omega_{1}, \quad(\mathbf{D} \nabla \mathrm{T}) \cdot \mathbf{n}=0 \text { on } \partial \Omega_{2}
$$

where $\partial \Omega_{1}$ is the heat sink, $\partial \Omega_{2}$ is the rest of the boundary and $\mathbf{n}$ is the outward normal to the boundary $\partial \Omega_{2}$.

The objective function is $c=\int_{\Omega} g T d \Omega$ for minimum thermal compliance problems and $c=$ $\left[\frac{1}{|\Omega|} \int_{\Omega} T^{p} d \Omega\right]^{\frac{1}{p}} \leq T_{\max }=\int_{\Omega} \delta T d \Omega$ for min-max temperature problems, where $\delta$ is the $\delta$-function supported at the point with the maximum temperature. When $p$ tends to infinity, $c$ approaches the real maximum temperature $T_{\max }$. Based on [33, 35, the optimization problems without material volume constraint is equivalent to

$$
I_{\mathrm{A}}=\min _{\rho, \mathbf{D}} \min _{T} \max _{\mu} \int_{\Omega}[F(T, \rho)-g \mu+\nabla \mu \cdot \mathbf{D} \nabla T]
$$

where, $\mu$ is the adjoint solution solving the following adjoint problem.

$$
\begin{gathered}
\nabla \cdot(\mathbf{D} \nabla \mu)=\frac{\partial F(T, \rho)}{\partial T} \text { in } \Omega \\
\mu=0 \text { on } \partial \Omega_{1}, \quad(\mathbf{D} \nabla \mu) \cdot \mathbf{n}=0 \text { on } \partial \Omega_{2}
\end{gathered}
$$

Consider a neighborhood of a point in the design domain. Assuming that the material density, the temperature field and adjoint solutions averaged over the neighborhood by the homogenization approach are fixed, Eq. (7) is reduced to [33, 35]

$$
\min _{\mathbf{D}} \nabla \mu \cdot \mathbf{D} \nabla T \geq J_{\mathrm{A}}=\|\nabla \mu\|\|\nabla T\|\left(\lambda_{\min } \cos ^{2} \theta-\lambda_{\max } \sin ^{2} \theta\right)
$$

with $\theta$ and $\lambda_{\min }, \lambda_{\max }$ defined as in Section 5. By utilizing the optimal rank-1 laminates, the optimization problem reduces to

$$
I_{\mathrm{A}}=\min _{\rho} \min _{T} \max _{\mu} \int_{\Omega}\left[F(T, \rho)-g \mu+J_{\mathrm{A}}\right]
$$

Considering the material volume constraint, the optimality criterion for material density distribu- 
tion is

$$
\frac{\partial}{\partial \rho}\left[F(T, \rho)-g \mu+J_{\mathrm{A}}\right]+\gamma=0
$$

where, $0<\rho<1, T$ and $\mu$ solve the primary and adjoint problems respectively, and $\gamma$ is the Lagrangian multiplier for material volume constraint.

In general, a numerical procedure is needed to solve the optimization problem Eq. (10), involving iterative computing of the temperature field and adjoint solution for a given material distribution and then finding the optimal distribution for fixed fields. However, for the 1D cases studied in this paper, the temperature field and adjoint solution can be expressed as explicit functions of the material distribution. The optimization problems thus reduce to standard minimization problems subject to an inequality constraint and bound constraints, hence can be solved analytically.

\section{A1.2 Rectangular volume-to-line structure}

The heat conduction equation and boundary conditions of the rectangular volume-to-line structure are

$$
\frac{d}{d x}\left(k \frac{d T}{d x}\right)+g=0, \quad T(x=0)=0,\left.\quad\left(k \frac{d T}{d x}\right)\right|_{x=L}=0
$$

where, $k$ is material conductivity. Since for the considered 1D cases the gradients of temperature and adjoint solution are anti-parallel to each other for both minimum thermal compliance and min-max temperature problems, material layers of optimal rank-1 laminates should direct along $x$-axis. Thus $k=\lambda_{\max }=\rho k_{0}+(1-\rho) k_{\min }$. Solving Eq. $(12)$, the temperature gradient field and temperature field are

$$
\left\{\begin{array}{l}
\frac{d T}{d x}=\frac{g}{k(x)}(L-x) \\
T(x)=\int_{0}^{x} \frac{g}{k(s)}(L-s) d s
\end{array}\right.
$$

The reduced optimization problem Eq. 10 for minimum thermal compliance objective then becomes

$$
I_{\mathrm{A}}=\min _{\rho} \int_{0}^{L} k\left(\frac{d T}{d x}\right)^{2} d x
$$

The corresponding optimality criterion is

$$
\frac{\partial}{\partial \rho}\left[k\left(\frac{d T}{d x}\right)^{2}\right]+\gamma=0
$$


The optimal material distribution can be computed,

$$
\rho_{0}= \begin{cases}1 & x \leq l_{1} \\ \frac{C}{k_{0}-k_{\min }}(L-x)-\frac{k_{\min }}{k_{0}-k_{\min }} & l_{1}<x<l_{2} \\ 0 & l_{2} \leq x \leq L\end{cases}
$$

where, $C=g \sqrt{\frac{k_{0}-k_{\min }}{\gamma}}$ and $l_{1}=L-\frac{k_{0}}{C}, l_{2}=L-\frac{k_{\min }}{C}$.

$$
\begin{gathered}
l_{1} \leq 0<l_{2} \\
\int_{0}^{l_{2}} \rho d x=f \int_{0}^{L} d x
\end{gathered}
$$

From Eq. 17, the relation between $f$ and $C$ is

$$
k_{\min }+f\left(k_{0}-k_{\min }\right)=\frac{1}{2}\left(L C+\frac{k_{\min }^{2}}{L C}\right)
$$

The range of $f$ is

$$
0<f \leq \frac{k_{0}-k_{\min }}{2 k_{0}}
$$

Taking the loading and boundary conditions of the rectangular volume-to-line structure into consideration, the maximum temperature is expected to appear at $x=L$. Hence, the reduced optimization problem Eq. (10) for min-max temperature objective is

$$
I_{\mathrm{A}}=\min _{\rho} \int_{0}^{L} \frac{g}{k}(L-x) d x
$$

The corresponding optimality criterion is

$$
\frac{\partial}{\partial \rho}\left[\frac{g}{k}(L-x)\right]+\gamma=0
$$


The optimal material distribution is

$$
\rho_{0}= \begin{cases}1 & x \leq l_{1} \\ \frac{C}{k_{0}-k_{\min }} \sqrt{L-x}-\frac{k_{\min }}{k_{0}-k_{\min }} & l_{1}<x<l_{2} \\ 0 & l_{2} \leq x \leq L\end{cases}
$$

where, $C=\sqrt{\frac{g\left(k_{0}-k_{\min }\right)}{\gamma}}$ and $l_{1}=L-\left(\frac{k_{0}}{C}\right)^{2}, l_{2}=L-\left(\frac{k_{\min }}{C}\right)^{2}$.

Assume no local region becomes fully solid in the optimal structure, the relation between $f$ and $C$

475 is then

$$
k_{\min }+f\left(k_{0}-k_{\min }\right)=\frac{2}{3} C L^{\frac{1}{2}}+\frac{k_{\min }^{3}}{3 C^{2} L}
$$

The range of $f$ is

$$
f\left(C L^{\frac{1}{2}}=k_{\min }\right)=0<f \leq f\left(C L^{\frac{1}{2}}=k_{0}\right)=\frac{\left(k_{0}-k_{\min }\right)\left(2 k_{0}+k_{\min }\right)}{3 k_{0}^{2}}
$$

\section{A1.3 Circular volume-to-line structure}

The heat conduction equation and boundary conditions of the circular volume-to-line structure are

$$
\frac{1}{r} \frac{d}{d r}\left(k r \frac{d T}{d r}\right)+g=0, \quad T\left(r=R_{\mathrm{s}}\right)=0,\left.\quad\left(k \frac{d T}{d r}\right)\right|_{r=R}=0
$$

The temperature gradient field and temperature field are

$$
\left\{\begin{array}{l}
\frac{d T}{d r}=\frac{g}{2 k}\left(\frac{R^{2}}{r}-r\right) \\
T(r)=\int_{R_{s}}^{r} \frac{g}{2 k(s)}\left(\frac{R^{2}}{s}-s\right) d s
\end{array}\right.
$$

Similar to the rectangular volume-to-line problem, the optimal material distribution for minimum thermal compliance is

$$
\rho_{0}= \begin{cases}1 & r \leq r_{1} \\ \frac{C}{k_{0}-k_{\min }} \frac{R^{2}-r^{2}}{r}-\frac{k_{\min }}{k_{0}-k_{\min }} & r_{1}<r<r_{2} \\ 0 & r_{2} \leq r \leq R\end{cases}
$$


where, $C=g \sqrt{\frac{k_{0}-k_{\min }}{4 \gamma}}$ and $r_{1}=\sqrt{R^{2}+\left(\frac{k_{0}}{2 C}\right)^{2}}-\frac{k_{0}}{2 C}, r_{2}=\sqrt{R^{2}+\left(\frac{k_{\min }}{2 C}\right)^{2}}-\frac{k_{\min }}{2 C}$.

The optimal material distribution for min-max temperature is

$$
\rho_{0}= \begin{cases}1 & r \leq r_{1} \\ \frac{C}{k_{0}-k_{\min }} \frac{\sqrt{R^{2}-r^{2}}}{r}-\frac{k_{\min }}{k_{0}-k_{\min }} & r_{1}<r<r_{2} \\ 0 & r_{2} \leq r \leq R\end{cases}
$$

485 where, $C=\sqrt{\frac{g\left(k_{0}-k_{\min }\right)}{2 \gamma}}$ and $r_{1}=\frac{C R}{\sqrt{C^{2}+k_{0}^{2}}}, r_{2}=\frac{C R}{\sqrt{C^{2}+k_{\min }^{2}}}$.

\section{Appendix A2. Effect of $p$-norm values for the min-max temperature problem}

To avoid solving a non-differentiable min-max problem with very many constraints, we use the aggregated $p$-norm temperature. A larger $p$ provides a more accurate approximation of the real maximum temperature, but increases the non-linearity of the objective function which could cause convergence problems. Hence, in Section 4 and Appendix A4, a value of $p=10$ is used.

To illustrate the effect of varying $p$ values, volume-to-point designs by the rank-1 laminates model for $p=1,10,20,40,60$ and corresponding temperature contour are plotted in Figure A2.1, as well as the design for minimum thermal compliance. The values of the temperature $p$-norm and the actual maximum temperature are also listed. For the optimization with $p>10$, the convergence condition is relaxed due to convergence problems. As the $p$-norm of temperature approximates the real maximum temperature more accurately, the temperature along the boundary away from the heat sink tends to equalize in the optimized designs, which is in line with the observations in [20. Since the real maximum temperature of the optimized design by using $p=10$ is only $3.7 \%$ larger than the design for $p=60$ and the optimized designs are virtually indistinguishable, it is in general reasonable to use $\|\mathbf{T}\|_{10}$ as the objective function to do min-max temperature optimization.

\section{Appendix A3. Comparison with other methods}

We claim that optimal heat conducting structures are needle- and not tree-like. In order to further substantiate this conclusion, we have compared our designs with their counterparts obtained 
Min thermal compliance

$$
T_{\max }=1.206 T_{0}
$$
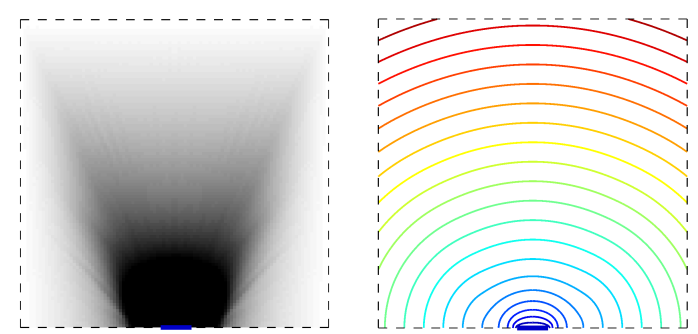

Min $\|\mathbf{T}\|_{10}$

$\|\mathbf{T}\|_{10}=0.874 T_{0}, T_{\max }=1.037 T_{0}$
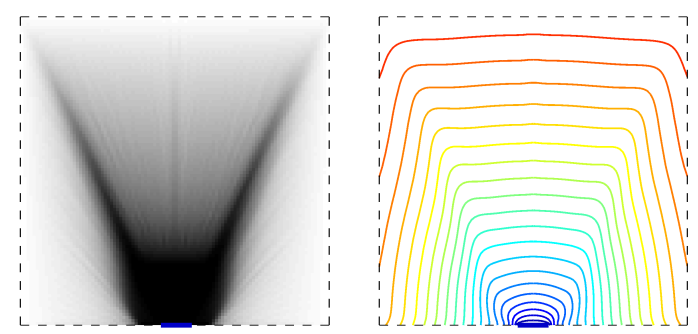

Min $\|\mathbf{T}\|_{40}$
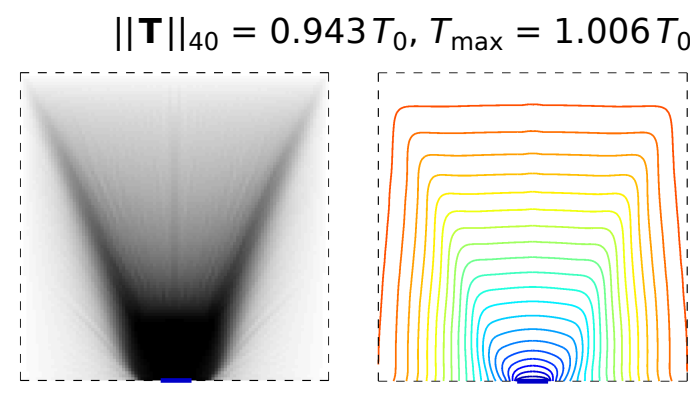$$
0
$$

Min $\|\mathbf{T}\|_{1}$

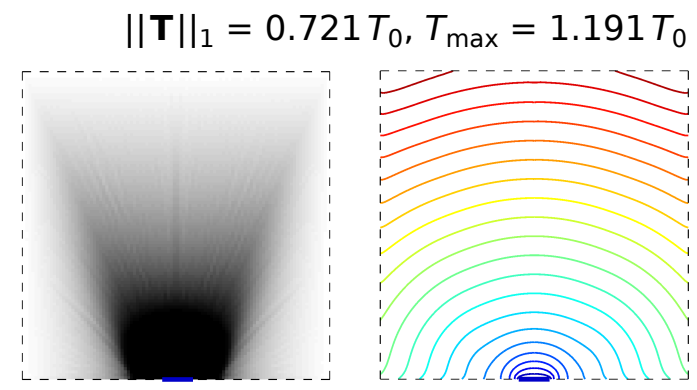

Min $\|\mathbf{T}\|_{20}$

\| $\mathbf{T} \|_{20}=0.914 T_{0}, T_{\max }=1.017 T_{0}$
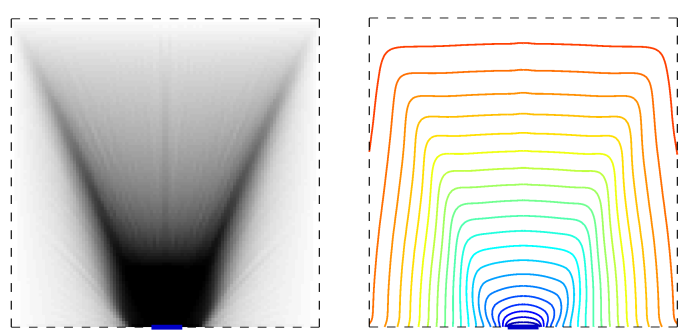

Min $\|\mathbf{T}\|_{60}$

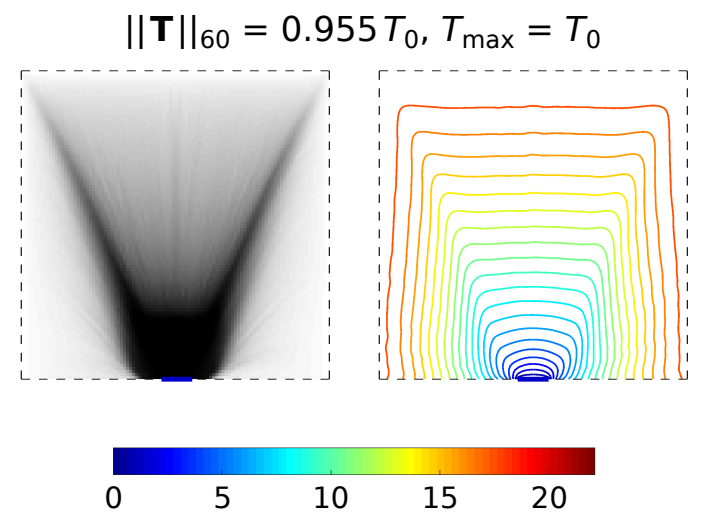

Figure A2.1: Rank-1 laminates designs for minimum thermal compliance and min-max temperature $\left(\|\mathbf{T}\|_{p}, p=\right.$ $1,10,20,40,60)$ and corresponding temperature contour, as well as the values of temerature $p$-norm and the real maximum temperature 
by other methods. Specifically, designs by genetic algorithms and data for the designs by cellular automaton and constructal approaches given in [20] are used for comparison.

We note that the volume-to-point optimization problem studied in 20 is design dependent, i.e. heat generation only happens in low conduction regions. Hence, the sensitivity analysis is updated accordingly. Only rank-1 laminates designs are involved in the comparison considering that no explicit minimum length scale is imposed in other methods and discrete black-white designs with nearly the same performance as rank-1 laminates designs can be readily obtained through projection as demonstrated in the main paper. Different ratios of material conductivities $k_{0} / k_{\min }=[2,10,50,100,200,250,400,600,800,1000]$ are used and the maximum allowable volume fraction of the high conductive material is restricted to be $f=0.3$. In the optimization for min-max temperature, the $p$-norm of temperature with $p=60$ is used as the objective function. Rank-1 designs for minimum mean temperature subject to design-independent and designdependent heat generation respectively are given in Figure A3.1, as well as corresponding designs from [20]. Optimized designs for min-max temperature are shown in Figure A3.2. As in [20], two non-dimensional thermal resistances $A$ and $R$ are used to assess the performance of minimum average temperature designs and min-max temperature designs, respectively. The non-dimensional parameters are defined as

$$
\begin{gathered}
A=\frac{T_{\text {mean }}-T_{\text {sink }}}{g L H / k_{\text {min }}} \\
R=\frac{T_{\max }-T_{\text {sink }}}{g L H / k_{\min }}
\end{gathered}
$$

where, $T_{\text {mean }}$ and $T_{\max }$ are the mean temperature and the maximum temperature of the optimized designs, respectively, $g$ is the heat generation rate per unit volume (surface), LH represents the area of the volume (surface), $k_{\min }$ is the conductivity of the low conductive material. The nondimensional parameter $R$ was first proposed in 1 to measure the performance of heat conducting structures under design-independent heat generation. However, when used for design-dependent problems as in 20 the above definitions of $A$ and $R$, ought to be scaled since the applied heat is reduced by a factor of $(1-f)$. However, to allow direct comparison with 20], Eqs. (29) and (30) are nevertheless used here for the rank-1 laminates designs under design-dependent heat generation, whereas for rank-1 laminates designs under design-independent heat generation, our obtained $A$ and $R$ parameters are scaled by $(1-f)$ for fair comparison. 
The $A$ and $R$ parameters for designs obtained by different methods are plotted in Figure A3.3 with respect to $\left(k_{0} / k_{\min }\right) f$. Red and blue symbols represent rank-1 laminates designs with designdependent and design-independent heat generation, respectively, and black symbols are data from 20] denoting designs by genetic algorithm, cellular automaton, constructal approaches and topology optimization methods using ESO algorithm and SIMP model, respectively. Symbols for the designs by the two constructal approaches are interchanged in Figure A3.3 compared to the graph in [20] after checking the original data from [46].

From Figure A3.3, we observe that rank-1 laminates designs (needle structures) perform significantly better than the designs (tree structures) obtained by the other methods. When the material conductivity contrast is small (e.g. $k_{0} / k_{\min }=2$ ), designs by the genetic algorithm have similar performance as corresponding rank-1 laminates designs. However, as the material contrast increases, designs by genetic algorithm become tree-like with more and more branches but worse and worse performance. When $k_{0} / k_{\min }=250$, the genetic algorithm design has a $39 \%$ larger objective than the corresponding rank-1 laminates design for minimum average temperature, and a $49 \%$ larger objective for the min-max temperature objective. The few cases where black points seem to be better than the our rank-1 results are points that use volume fractions different from $f=0.3$ and hence should have been differently scaled.

In [20], parameters $A$ and $R$ are expressed as functions of $\left(k_{0} / k_{\min }\right) f$

$$
\begin{aligned}
& \log _{10}\left(A_{\mathrm{GA}}\right)=-0.8572 \log _{10}\left[\left(k_{0} / k_{\min }\right) f\right]-0.7331 \\
& \log _{10}\left(R_{\mathrm{GA}}\right)=-0.8428 \log _{10}\left[\left(k_{0} / k_{\min }\right) f\right]-0.5528
\end{aligned}
$$

The corresponding expressions for our rank-1 laminates designs with $f=0.3$ are

$$
\begin{aligned}
& \log _{10}\left(A_{\text {rank-1 }}\right)=-0.03337\left\{\log _{10}\left[\left(k_{0} / k_{\min }\right) f\right]\right\}^{2}-0.8712 \log _{10}\left[\left(k_{0} / k_{\min }\right) f\right]-0.7288 \\
& \log _{10}\left(R_{\text {rank-1 }}\right)=-0.0469\left\{\log _{10}\left[\left(k_{0} / k_{\text {min }}\right) f\right]\right\}^{2}-0.8331 \log _{10}\left[\left(k_{0} / k_{\min }\right) f\right]-0.5764
\end{aligned}
$$


design-independent heat generation are even better in terms of $A$ and $R$ parameters

$$
\begin{aligned}
& \log _{10}\left(A_{\text {rank-1 }}^{\mathrm{In}}\right)=-0.03337\left\{\log _{10}\left[\left(k_{0} / k_{\min }\right) f\right]\right\}^{2}-0.8712 \log _{10}\left[\left(k_{0} / k_{\min }\right) f\right]-0.7622 \\
& \log _{10}\left(R_{\text {rank-1 }}^{\mathrm{In}}\right)=-0.0469\left\{\log _{10}\left[\left(k_{0} / k_{\min }\right) f\right]\right\}^{2}-0.8331 \log _{10}\left[\left(k_{0} / k_{\min }\right) f\right]-0.5976
\end{aligned}
$$

In both cases, our functions almost coincide with those obtained in 20] in the low contrast limit (where influence of topology becomes less and less important), whereas they provide much lower estimates for higher conductivity contrasts.

At the end we note that we have conducted optimization using different $\left(k_{0} / k_{\min }, f\right)$ pairs but the same $\left(k_{0} / k_{\min }\right) f$ products. The derivation in $A$ and $R$ parameters can be up to $40 \%$ for the same $\left(k_{0} / k_{\min }\right) f$ product. Hence the $A$ and $R$ parameters for rank-1 laminates designs with $\left(k_{0} / k_{\min }, f\right)=(10,0.1),(10,0.5)$ are also plotted in Figure A3.3 for fair comparison with genetic algorithm designs obtained for the same parameter value. $f=0.1$ is used when comparing with the first design by the cellular automaton method in Figure A3.3.

\section{Appendix A4. Approaches to improve convergence of density approaches}

\section{A4.1 Continuation approach}

Minimum thermal compliance problems under single load conditions are convex when densitybased material models without penalization like the variable thickness sheet model are used. As soon as a penalization on intermediate densities is applied, the problems become non-convex and prone to converging to local minima. Continuation approaches are often advisable to obtain a better local minimum where the penalization is increased slowly with the optimization process [47]. Hence, a series of optimization problems are solved starting from the non-penalized, convex problem ending at the one with the prescribed penalization. The design result of the previous optimization problem is used as the initial guess for the following problem. From Wadbro and Berggren's work [13, we observe that when a fine mesh, a strict convergence condition (and a slow continuation approach) are used, hair-like designs with no obvious branches are obtained by the SIMP model in the optimization of a volume-to-point structure for minimum thermal compliance purpose.

In the following, the volume-to-point structure in Section 3 is optimized by the SIMP and RAMP models respectively using a uniform initial guess to verify the ability of penalized models in achieving (near-)optimal designs. A discretization of $800 \times 800$ elements is used and the filter radius of the 

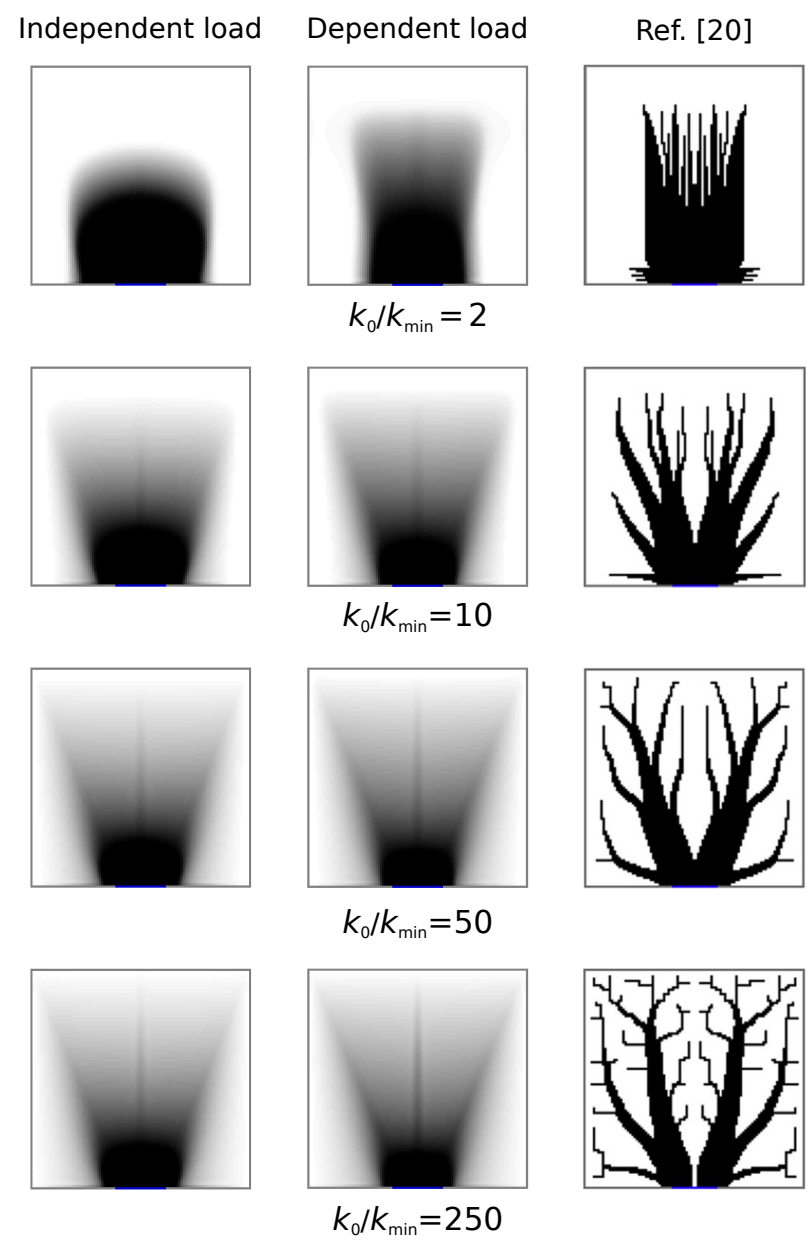

(b)

(c)

Figure A3.1: Rank-1 laminates designs subject to (a) design-independent, (b) design-dependent heat generation and (c) designs from 20] for minimum mean temperature $(f=0.3)$ 

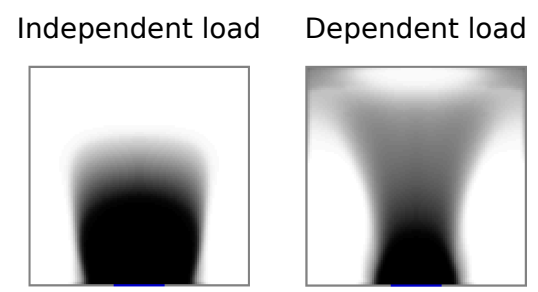

Ref. [20]
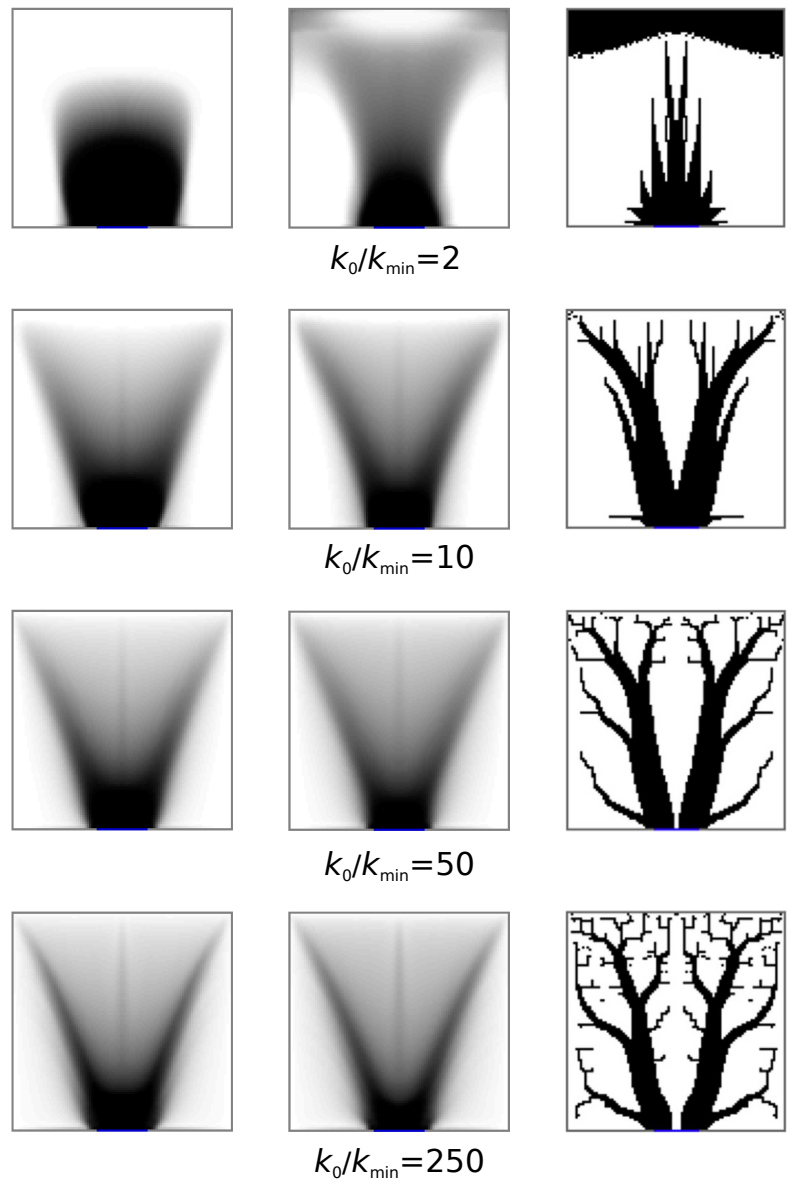

(a)

(b)

(c)

Figure A3.2: Rank-1 laminates designs subject to (a) design-independent, (b) design-dependent heat generation and (c) designs from [20] for min-max temperature $(f=0.3)$ 


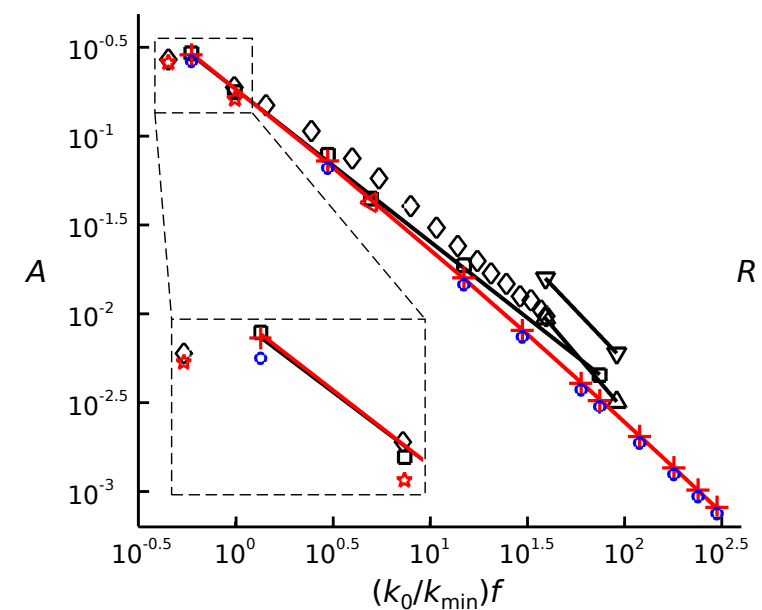

$\diamond$ Cellular automaton

口 Genetic algorithm

Fit for genetic algorithm

$\rightarrow$ ESO algorithm

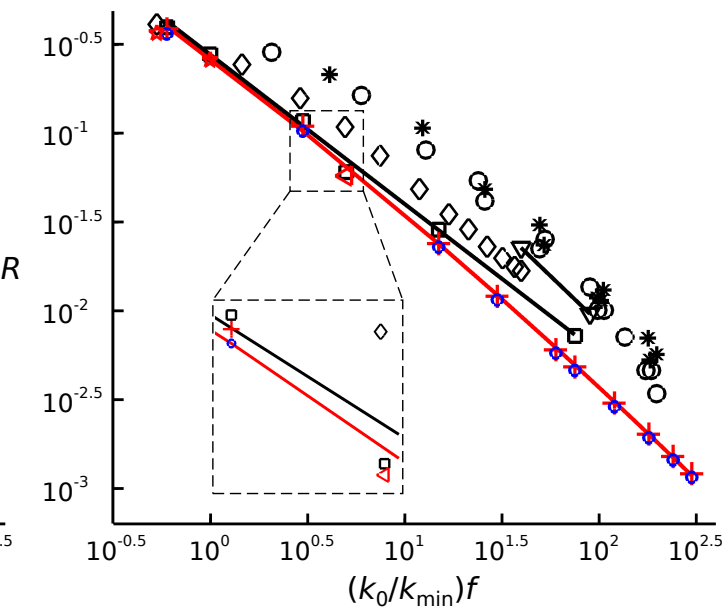

* Constructal, Bejan

- Constructal, Ghodossi

- Rank-1 model, $f=0.3$

(Independent heat genertaion)
+ Rank-1 model, $f=0.3$

ش Rank-1 model, $f=0.1$

$\triangleleft$ Rank-1 model, $f=0.5$
Fit for rank-1 model, $f=0.3$

Figure A3.3: Comparison of designs obtained by different methods in terms of $A$ and $R$ parameters. Black symbols and curves are from [20] and colored symbols and curves are from the current work. Design-dependent heat generation is used for all the designs, except for the ones represented by blue symbols. Data of blue symbols are scaled according to the generated heat 
density filter is $R_{\min }=\frac{1.1 \mathrm{~L}}{800}$. When the maximum change of design variables between iterations is smaller than 0.001 , the optimization is considered as converged. Two continuation approaches are used. With the first continuation approach, the penalization parameter is increased in steps of $\Delta q=0.5$ between optimization rounds up to the maximum value. In the second approach, the penalization parameter is increased in the same way as in the first approach except that $\Delta q$ is increased tenfold every 3000 iterations from $5 \times 10^{-6}$ to 0.5 . Therefore, the penalization on intermediate densities is quite small at the beginning of the optimization when using the second continuation approach. A maximum penalization of 3 is used for the SIMP model and 4 for the RAMP model. The optimality criterion method [26] is adopted to solve the optimization problem. After the whole optimization procedure converges, design results are projected into strict black-white, while preserving the volume of high conductive material. Design results with and without continuation are given in Figure A4.1. The effect of the continuation approach on the non-self adjoint min-max temperature problem is also studied. The RAMP model is used for material interpolation and MMA as the optimizer. Considering the nonlinearity of the problem, the convergence tolerance is relaxed to 0.003 . Other parameters are the same as the above minimum thermal compliance problem. Designs from using a fixed penalization and the second continuation approach are given in Figure A4.2.

As illustrated by the design results, (near-)optimal designs with no obvious branches can also be obtained by penalized density-based material models, when a slow continuation approach is used. Although Wadbro and Berggren obtained hair-like designs even without a slow continuation approach probably due to the single precision accuracy of GPUs, our work shows that a slow increase of penalization is quite important especially in the early stage of optimization. Resulting from the zero derivative at zero material density, the design obtained from the SIMP model without continuation has the worst performance.

\section{A4.2 Local volume constraint}

Enforcing local volume constraints [48, 49] could be another potential way for penalized densitybased material models to converge to better designs. To restrict local material accumulation, the following additional volume constraint is added to the original optimization problem Eq. (6).

$$
\left[\frac{1}{n} \sum_{e=1}^{n}\left(\bar{\rho}_{e}\right)^{P}\right]^{\frac{1}{P}} \leq \varepsilon
$$




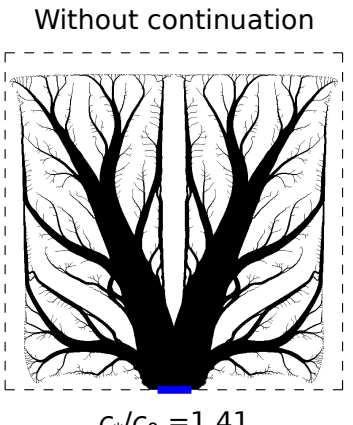

$C * / c_{0}=1.41$

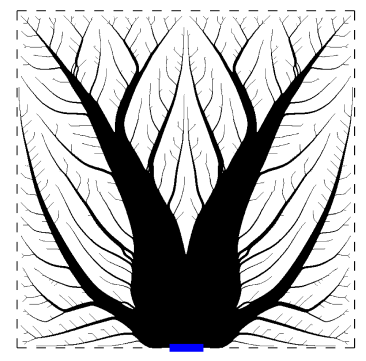

$C * / c_{0}=1.14$

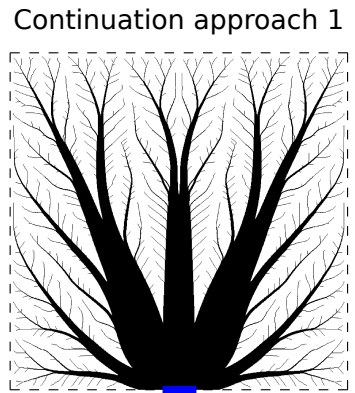

$C * / c_{0}=1.10$

(a)

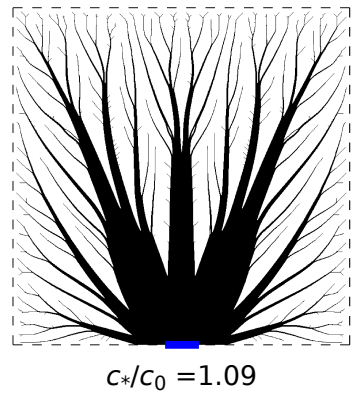

(b)

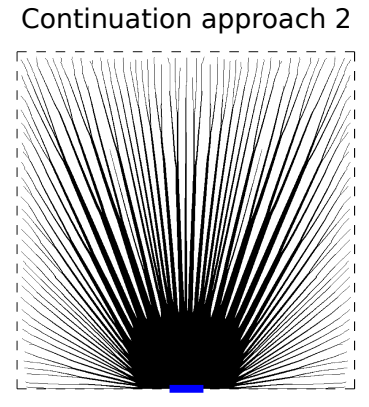

$C * / C_{0}=1.06$

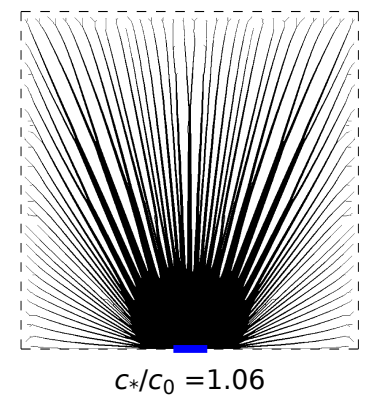

Figure A4.1: Designs of the volume-to-point structure for minimum thermal compliance by (a) SIMP model, and (b) RAMP model and objective function ratios compared to the corresponding rank-1 laminates design

Without continuation

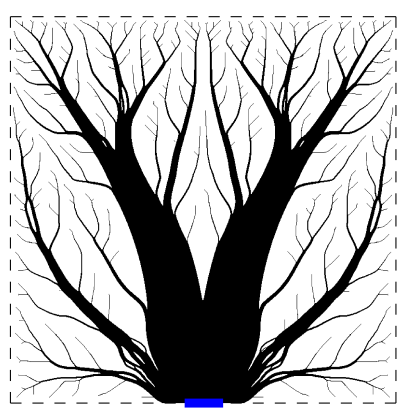

$C * / C_{\mathrm{r} 1}=1.13, T_{*} / T_{\mathrm{r} 1}=1.16$
Continuation approach 2

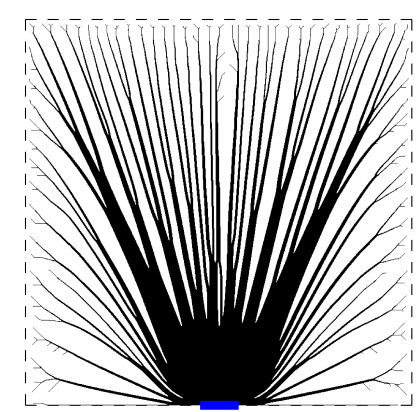

$C_{*} / C_{\mathrm{r} 1}=1.08, T_{*} / T_{\mathrm{r} 1}=1.13$

Figure A4.2: Designs of the volume-to-point structure for min-max temperature and ratios compared to the corresponding rank-1 laminates design 
where, $\bar{\rho}_{e}=\sum_{i \in \mathbb{N}_{e}} \rho_{i} / \sum_{i \in \mathbb{N}_{e}} 1$ measures the local material volume fraction over the neighborhood $\mathbb{N}_{e}$ of element $e, \mathbb{N}_{e}=\left\{i \mid\left\|x_{i}-x_{e}\right\|_{2} \leq R_{\mathrm{v}}\right\}$ with $x_{i}$ and $x_{e}$ denoting the centroids of elements $i$ and $e$ respectively and $R_{\mathrm{v}}$ is a prescribed influence radius, $n$ is the total number of elements, $P$ is the $p$-norm value and $\varepsilon$ is the prescribed upper bound.

The new volume-to-point problems incorporating the local volume constraint Eq. (34) are solved starting from a uniform initial guess. The RAMP model is used with a penalization $q=4$ and MMA is the optimizer. Other parameters are set as $P=8, R_{\mathrm{v}}=L / 60, n=800 \times 800, \varepsilon=0.5$. The local volume constraint is switched off after 200 iterations. The optimization terminates when the maximum design variable change between iterations is smaller than 0.01 or the number of iterations reaches 1000 . Then designs are projected into strict black-white while preserving the volume constraint. As shown in Figure A4.3, large material blocks on diagonals are avoided and design results tend to be needle-like. Suboptimal topological features, i.e. wavy needles and inner holes, are observed as in Wadbro and Berggren's designs [13. An alternative method of imposing local volume constraints is based on rank-1 designs by constraining the local volume difference between optimized designs and corresponding rank-1 designs. In addition, when a minimum length scale is also imposed, its relation with the local volume constraint requires extra attention to obtain satisfying designs.

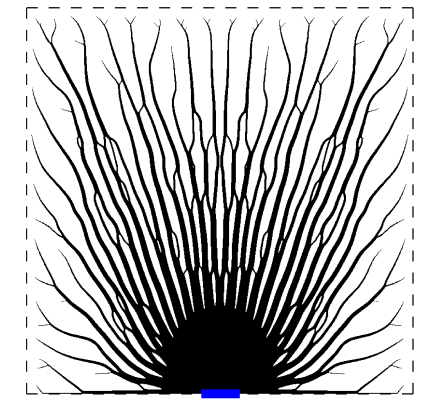

$c_{*} / c_{\mathrm{r} 1}=1.13$

(a)

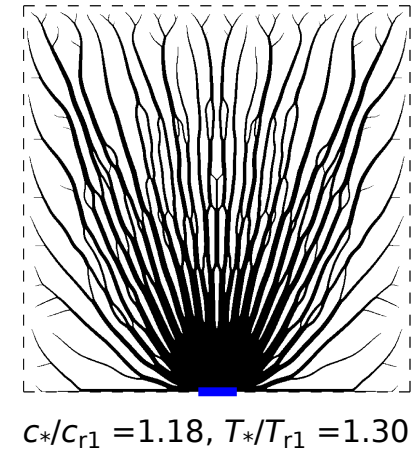

(b)

Figure A4.3: Designs of the volume-to-point structure with a local volume constraint for (a) minimum thermal compliance, and (b) min-max temperature and ratios compared to the corresponding rank-1 laminates designs

\section{References}

[1] A. Bejan, Constructal-theory network of conducting paths for cooling a heat generating volume, International Journal of Heat and Mass Transfer 40 (4) (1997) 799-816. 
[2] L. Rocha, S. Lorente, A. Bejan, Constructal design for cooling a disc-shaped area by conduction, International Journal of Heat and Mass Transfer 45 (8) (2002) 1643-1652.

[3] A. Bejan, Shape and structure, from engineering to nature, Cambridge university press, 2000.

[18] B. Li, J. Hong, X. Tian, Generating optimal topologies for heat conduction by heat flow paths identification, International Communications in Heat and Mass Transfer 75 (2016) 177-182.

[19] K. Guo, W. Qi, B. Liu, C. Liu, Z. Huang, G. Zhu, Optimization of an area to point heat conduction problem, Applied Thermal Engineering 93 (2016) 61-71. of Applied Physics 93 (8) (2003) 4922-4929.

[5] L. Ghodoossi, Conceptual study on constructal theory, Energy conversion and management 45 (9) (2004) 1379-1395.

[6] L. Ghodoossi, Entropy generation rate in uniform heat generating area cooled by conducting paths: criterion for rating the performance of constructal designs, Energy conversion and management 45 (18) (2004) 2951-2969.

[7] G. Marck, J.-L. Harion, M. Nemer, S. Russeil, D. Bougeard, A new perspective of constructal networks cooling a finite-size volume generating heat, Energy Conversion and Management 52 (2) (2011) 10331046 .

[8] M. C. E. Manuel, P. T. Lin, Design explorations of heat conductive pathways, International Journal of Heat and Mass Transfer 104 (2017) 835-851.

[9] J. Dirker, J. P. Meyer, Topology optimization for an internal heat-conduction cooling scheme in a square domain for high heat flux applications, Journal of Heat Transfer 135 (11) (2013) 111010.

[10] G. Marck, M. Nemer, J.-L. Harion, S. Russeil, D. Bougeard, Topology optimization using the simp method for multiobjective conductive problems, Numerical Heat Transfer, Part B: Fundamentals 61 (6) (2012) 439-470.

[11] A. Gersborg-Hansen, M. P. Bendsøe, O. Sigmund, Topology optimization of heat conduction problems using the finite volume method, Structural and multidisciplinary optimization 31 (4) (2006) 251-259.

[12] J. K. Guest, Topology optimization with multiple phase projection, Computer Methods in Applied Mechanics and Engineering 199 (1) (2009) 123-135.

[13] E. Wadbro, M. Berggren, Megapixel topology optimization on a graphics processing unit, SIAM review 51 (4) (2009) 707-721.

[14] T. Yamada, K. Izui, S. Nishiwaki, A level set-based topology optimization method for maximizing thermal diffusivity in problems including design-dependent effects, Journal of Mechanical Design 133 (3) (2011) 031011.

[15] E. M. Dede, Simulation and optimization of heat flow via anisotropic material thermal conductivity, Computational Materials Science 50 (2) (2010) 510-515.

[16] Y. Li, P. Wei, H. Ma, Integrated optimization of heat-transfer systems consisting of discrete thermal conductors and solid material, International Journal of Heat and Mass Transfer 113 (2017) 1059-1069.

[17] Y. Zhang, S. Liu, The optimization model of the heat conduction structure, Progress in Natural Science 18 (6) (2008) 665-670.

[4] L. Ghodoossi, N. Eğrican, Exact solution for cooling of electronics using constructal theory, Journal 
[38] M. Rossow, J. Taylor, A finite element method for the optimal design of variable thickness sheets, Aiaa Journal 11 (11) (1973) 1566-1569.

20] R. Boichot, Y. Fan, A genetic algorithm for topology optimization of area-to-point heat conduction problem, International Journal of Thermal Sciences 108 (2016) 209-217.

[21] X. Xu, X. Liang, J. Ren, Optimization of heat conduction using combinatorial optimization algorithms, International journal of heat and mass transfer 50 (9) (2007) 1675-1682.

[22] R. Boichot, L. Luo, Y. Fan, Tree-network structure generation for heat conduction by cellular automaton, Energy Conversion and Management 50 (2) (2009) 376-386.

[23] B. Li, J. Hong, S. Yan, H. Liu, L. Ge, Generating optimal heat conduction paths based on bionic growth simulation, International Communications in Heat and Mass Transfer 83 (2017) 55-63.

[24] D. J. Lohan, E. M. Dede, J. T. Allison, Topology optimization for heat conduction using generative design algorithms, Structural and Multidisciplinary Optimization 55 (3) (2017) 1063-1077.

[25] H. Pedro, M. H. Kobayashi, C. F. Coimbra, A. de Silva, Effectiveness of complex design through an evolutionary approach, Journal of Thermophysics and Heat Transfer 22 (1) (2008) 115.

[26] M. P. Bendsøe, O. Sigmund, Topology optimization by distribution of isotropic material, Springer, 2004.

[27] M. P. Bendsøe, Optimal shape design as a material distribution problem, Structural and multidisciplinary optimization 1 (4) (1989) 193-202.

[28] G. I. Rozvany, M. Zhou, T. Birker, Generalized shape optimization without homogenization, Structural and Multidisciplinary Optimization 4 (3) (1992) 250-252.

[29] M. Stolpe, K. Svanberg, An alternative interpolation scheme for minimum compliance topology optimization, Structural and Multidisciplinary Optimization 22 (2) (2001) 116-124.

[30] C. D. Murray, The physiological principle of minimum work applied to the angle of branching of arteries, The Journal of general physiology 9 (6) (1926) 835.

[31] E. Katifori, G. J. Szöllősi, M. O. Magnasco, Damage and fluctuations induce loops in optimal transport networks, Physical Review Letters 104 (4) (2010) 048704.

[32] F. Corson, Fluctuations and redundancy in optimal transport networks, Physical Review Letters 104 (4) (2010) 048703.

[33] A. Cherkaev, Relaxation of problems of optimal structural design, International journal of solids and structures 31 (16) (1994) 2251-2280.

[34] G. Allaire, Shape Optimization by the Homogenization Method, Springer, New York Berlin Heidelberg, 2002.

[35] A. Cherkaev, Variational methods for structural optimization, Vol. 140, Springer Science \& Business Media, 2012.

[36] O. Sigmund, N. Aage, E. Andreassen, On the (non-) optimality of michell structures, Structural and Multidisciplinary Optimization 54 (2) (2016) 361-373.

[37] K. Svanberg, The method of moving asymptotesa new method for structural optimization, International journal for numerical methods in engineering 24 (2) (1987) 359-373. 
[39] M. P. Bendsøe, O. Sigmund, Material interpolation schemes in topology optimization, Archive of applied mechanics 69 (9) (1999) 635-654.

[40] B. Bourdin, Filters in topology optimization, International Journal for Numerical Methods in Engineering 50 (9) (2001) 2143-2158.

[41] T. E. Bruns, D. A. Tortorelli, Topology optimization of non-linear elastic structures and compliant mechanisms, Computer Methods in Applied Mechanics and Engineering 190 (26) (2001) 3443-3459.

[42] F. Wang, B. S. Lazarov, O. Sigmund, On projection methods, convergence and robust formulations in topology optimization, Structural and Multidisciplinary Optimization 43 (6) (2011) 767-784.

[43] X. Qian, O. Sigmund, Topological design of electromechanical actuators with robustness toward overand under-etching, Computer Methods in Applied Mechanics and Engineering 253 (2013) 237-251.

[44] J. P. Groen, O. Sigmund, Homogenization-based topology optimization for high-resolution manufacturable microstructures, International Journal for Numerical Methods in Engineeringdoi:10.1002/ nme.5575

[45] M. P. Bendsøe, N. Kikuchi, Generating optimal topologies in structural design using a homogenization method, Computer methods in applied mechanics and engineering 71 (2) (1988) 197-224.

[46] G. Marck, Topology optimization of heat and mass transfer: application to heat exchangers, Ph.D. thesis, Mines ParisTech University (12 2012).

[47] O. Sigmund, K. Maute, Topology optimization approaches, Structural and Multidisciplinary Optimization 48 (6) (2013) 1031-1055.

[48] J. Wu, N. Aage, R. Westermann, O. Sigmund, Infill optimization for additive manufacturing - approaching bone-like porous structures, IEEE Transactions on Visualization and Computer Graphics 24 (2) (2018) 1127-1140.

[49] B. S. Lazarov, F. Wang, Maximum length scale in density based topology optimization, Computer Methods in Applied Mechanics and Engineering 318 (2017) 826-844. 